\title{
Fuzzy Dynamic Parameter Adaptation in the Bird Swarm Algorithm for Neural Network Optimization
}

Patricia Melin ( $\nabla$ pmelin@tectijuana.mx )

Tijuana Institute of Technology https://orcid.org/0000-0001-5798-1426

Ivette Miramontes

Tijuana Institute of Technology

\section{Oscar Carvajal}

Tijuana Institute of Technology

\section{German Prado-Arechiga}

Excell Medical Center

\section{Research Article}

Keywords: Blood Pressure, Hypertension, Optimization, Fuzzy System, Bird Swarm Algorithm (BSA)

Posted Date: July 16th, 2021

DOI: https://doi.org/10.21203/rs.3.rs-694178/v1

License: (c) (i) This work is licensed under a Creative Commons Attribution 4.0 International License.

Read Full License

Version of Record: A version of this preprint was published at Soft Computing on January 10th, 2022. See the published version at https://doi.org/10.1007/s00500-021-06729-7. 


\title{
Fuzzy Dynamic Parameter Adaptation in the Bird Swarm Algorithm for Neural Network Optimization
}

\author{
*Patricia Melin ${ }^{1}$, Ivette Miramontes ${ }^{1}$, Oscar Carvajal ${ }^{1}$, German Prado-Arechiga ${ }^{2}$ \\ ${ }^{1}$ Tijuana Institute of Technology, Tijuana, Mexico \\ ${ }^{2}$ Cardiodiagnostico Excel Medical Center, Tijuana, Mexico \\ *Corresponding Author Email: pmelin@ @ectijuana.mx
}

\begin{abstract}
Fuzzy dynamic parameter adaptation has proven to be of great help when it is implemented in bioinspired algorithms for optimization in different application areas, such as control, mathematical functions, classification, among others. One of the main contributions of this work is the proposed improvement of the Bird Swarm algorithm using a Fuzzy System approach, and we called this improvement the Fuzzy Bird Swarm Algorithm. Furthermore, we use a set of complex Benchmark Functions of the Congress on Evolutionary Computation Competition 2017 to compare the results between the original algorithm and the proposed improvement of the algorithm. The fuzzy system is utilized for the dynamic parameter adaptation of $\mathrm{C} 1$ and $\mathrm{C} 2$ parameters of the Bird Swarm Algorithm. As a result, the Fuzzy Bird Swarm Algorithm has enhanced exploration and exploitation abilities that help in achieving better results than the Bird Swarm Algorithm. We additionally test the algorithm's performance in a real problem in the medical area, using the optimization of a neural network to obtain the risk of developing hypertension. This neural network uses patient information, such as age, gender, body mass index, systolic pressure, diastolic pressure, if the patient smokes and if the patient has parents with hypertension. Hypertension is one of the leading causes of heart problems, which in turn are also the top causes of death. Moreover, these days it causes more complications and deaths in people infected with COVID-19, the virus of the ongoing pandemic. Based on the results obtained through the 30 experiments carried out in three different study cases, and the results obtained from the statistical tests, it can be concluded that the proposed method provides better performance when compared with the original method.
\end{abstract}

Keywords: Blood Pressure, Hypertension, Optimization, Fuzzy System, Bird Swarm Algorithm (BSA).

\section{Introduction}

Bio-inspired algorithms have been very useful in solving optimization problems [1]-[5]. In addition, to further improve these results, dynamic parameter adaptation has been used, and these adaptations can be of different types [6]-[10]. 
Dynamic parameter adaptation using fuzzy inference systems have been used in different metaheuristics, such as Particle Swarm Optimization [11], Harmony Search Algorithm [12], Differential Evolution Algorithm [13], Galactic Swarm Optimization [14], just to name a few. The Bird Swarm Algorithm (BSA) was previously utilized for neural network optimization [15]. The results suggest that the BSA can be modified to achieve better results and this motivated this work. The novelty of this work is the improvement of the BSA by using Fuzzy Dynamic Parameter Adaptation of the Cognitive (C1) and the Social (C2) acceleration coefficients, which helps the algorithm with exploration and exploitation in the search space. Bird Swarm Algorithm was originally proposed by Meng in 2015 [16], mainly inspired by two behaviors of birds: social behavior

For the proposed method, we first studied which of the parameters, when changed produces a greater difference in the results. In this case, the parameters are the coefficients of cognitive, and social acceleration, respectively. Then three different fuzzy systems are created for which the rules are varied, using them in an ascending and descending way in addition to conducting experimentation with Trapezoidal and Gaussian membership functions to observe with which of these two, variants the better results are obtained.

In the experimental part, three case studies are carried out, in the first experiments with 10 classical mathematical functions; here as a disadvantage, it was not possible to differentiate which of the fuzzy systems provided the best result, since results were similar. For the second case study, 10 mathematical functions of the Congress on Evolutionary Computation 2017 (CEC2017) competition [17], [18] are used. In the third case, and most important case study, the optimization of the neural network architecture is carried out, in these two cases if a difference is observed in the fuzzy systems that provide better results. In addition, that when 
comparing the results obtained with the original algorithm if there is a significant improvement.

The main contribution presented in this work is to use the ability to manage uncertainty of fuzzy systems to perform dynamic parameter adaptation to the BSA algorithm in order to improve its performance, since it has been shown that by modifying bio-inspired algorithms in this way they have presented significant improvements in the results provided. The proposed method was used to optimize the architecture of an artificial neural network since it is part of a neuro-fuzzy model for obtaining a medical diagnosis based on blood pressure because the neural network should be able to produce accurate results.

This paper is structured as follows: Section 2 introduces the basic concepts, in Section 3, related works are described, in Section 4 the problem statement and proposed method are disclosed, in Section 5 the results of different experiments and the discussion are presented, and finally in Section 6 the conclusions and future work are outlined.

\section{Basic Concepts}

\subsection{Bird Swarm Algorithm}

The Bird Swarm Algorithm (BSA) [16], is inspired by studying the behavior of birds in the swarm. These behaviors are social behavior, social interaction, mimic feeding, flying, and vigilance, which can be used in solving optimization problems.

BSA is relatively new, it was proposed in 2015 by Meng. In this case, the social behaviors of birds are synthesized in five rules listed below: 
1- The behaviors of vigilance and foraging may switch in each bird. The two aforementioned behaviors are modeled as stochastic decisions.

2- Whereas in foraging, birds can record and update the best previous experience of each, and the swarms best previous experience regarding patch of food. The experience mentioned above can be used to search for food. Regarding social information, this is shared instantly between the complete swarm.

Rule 3: While vigilance is kept, all birds aspire to move to the middle of the swarm; this action can be affected by the interference provoked by the competition in the entire swarm. The Birds with the higher provisions will have a higher possibility of lying proximal to the center of the swarm than birds with low food reserves.

Rule 4: Birds habitually travel to different sites; birds can switch in producing and scrounging when this is done. The bird with the highest food reserve would be a producer, and the one with the lowest provisions would be a scrounger. Birds with intermediate provisions would randomly select to be a producer and scrounger.

Rule 5: Producers actively search for food. Scroungers would randomly follow a producer to search for food.

Mathematically, the previous rules can be analyzed as:

a) Foraging behavior

For this, while each bird is looking for food based on its experience and the swarm's experience, it is expressed by: 


$$
\begin{aligned}
x_{i, j}^{t+1}=x_{i, j}^{t}+ & \left(p_{i, j}-x_{i, j}^{t}\right) \times C X \times \operatorname{rand}(0,1)+\left(g_{j}-x_{i, j}^{t}\right) \times S \\
& \times \operatorname{rand}(0,1),
\end{aligned}
$$

where $j \in[1, \ldots, D]$, rand $(0,1)$ are independent numbers uniformly distributed in the range of $(0,1)$.

$C$ and $S$ can be called cognitive and social acceleration coefficients respectively and which each one represents two positive numbers. $P_{i, j}$ represents the best previous position in the $i_{\text {th }}$ bird and $g_{j}$ represents the best previous position in the swarm.

b) Vigilance behavior,

In this part, all the birds would attempt to move to the middle of the swarm and compete with others; for this, any bird would not move in a direct way to the center of the swarm; this is represented as follows:

$$
\begin{aligned}
& x_{i, j}^{t+1}=x_{i, j}^{t}+ A 1\left(\text { mean }_{j}-x_{i, j}^{t}\right) \times \operatorname{rand}(0,1)+A 2\left(p_{k, j}-x_{i, j}^{t}\right) \\
& \times \operatorname{rand}(-1,1) \\
& A 1=a 1 \times \exp \left(-\frac{p F i t_{i}}{\text { sumFit }+\varepsilon_{1}} \times N\right) \\
& A 2=a 2 \times \exp \left(\left(\frac{p F i t_{i}-p F i t_{k}}{\left|p F i t_{k}-p F i t_{i}\right|+\varepsilon}\right) \frac{N \times p F i t_{k}}{\text { sumFit }+\varepsilon}\right)
\end{aligned}
$$

In this expression, $k$ is a positive integer randomly selected between 1 and $N . p$ Fit $_{i}$ denotes the best fitness value in the $i$ th position and sumFit corresponds to the sum of the best fitness value of the swarms. $\varepsilon$ is a small value which helps to avoid the error in zero-division. mean $_{j}$ is the $j$ th element of the average place of the entire swarm. $a_{1}$ and $a_{2}$ represent positive constants in $[0,2]$. 
Given that all the birds aspire to occupy the center of the swarm, the product of $A 1$ and rand $(0,1)$ could not be more than 1 . A2 is utilized to simulate the direct effect produced by an interference when a bird moves towards the center of the swarm.

\section{c) Flight Behavior}

Birds may travel to another place in response to predation threats, foraging, or any different situation. Once they are moved to a different area, the birds will look for food again. The producing birds search for food patches, whereas the scroungers attempt to feed on the food patch discovered by the producers. Mathematically these behaviors be can be analyzed as:

$$
\begin{gathered}
x_{i, j}^{t+1}=x_{i, j}^{t}+\operatorname{rand} n(0,1) \times x_{i, j}^{t}, \\
x_{i, j}^{t+1}=x_{i, j}^{t}+\left(x_{k, j}^{t}-x_{i, j}^{t}\right) \times F L \times \operatorname{rand}(0,1),
\end{gathered}
$$

In these expressions, randn $(0,1)$ represents Gaussian distributed random numbers with mean 0 and standard deviation $1, \mathrm{k} \in[1,2,3 \ldots \mathrm{N}], \mathrm{k} \neq \mathrm{i}$. FL $(\mathrm{FL} \in[0,2])$ expresses that the scrounger will follow the producer to look for food

For simplification, Meng assumes that any bird can travel to another site every FQ unit interval, where $\mathrm{FQ}$ is a positive integer.

The pseudo-code of BSA is illustrated in Fig. 1. 


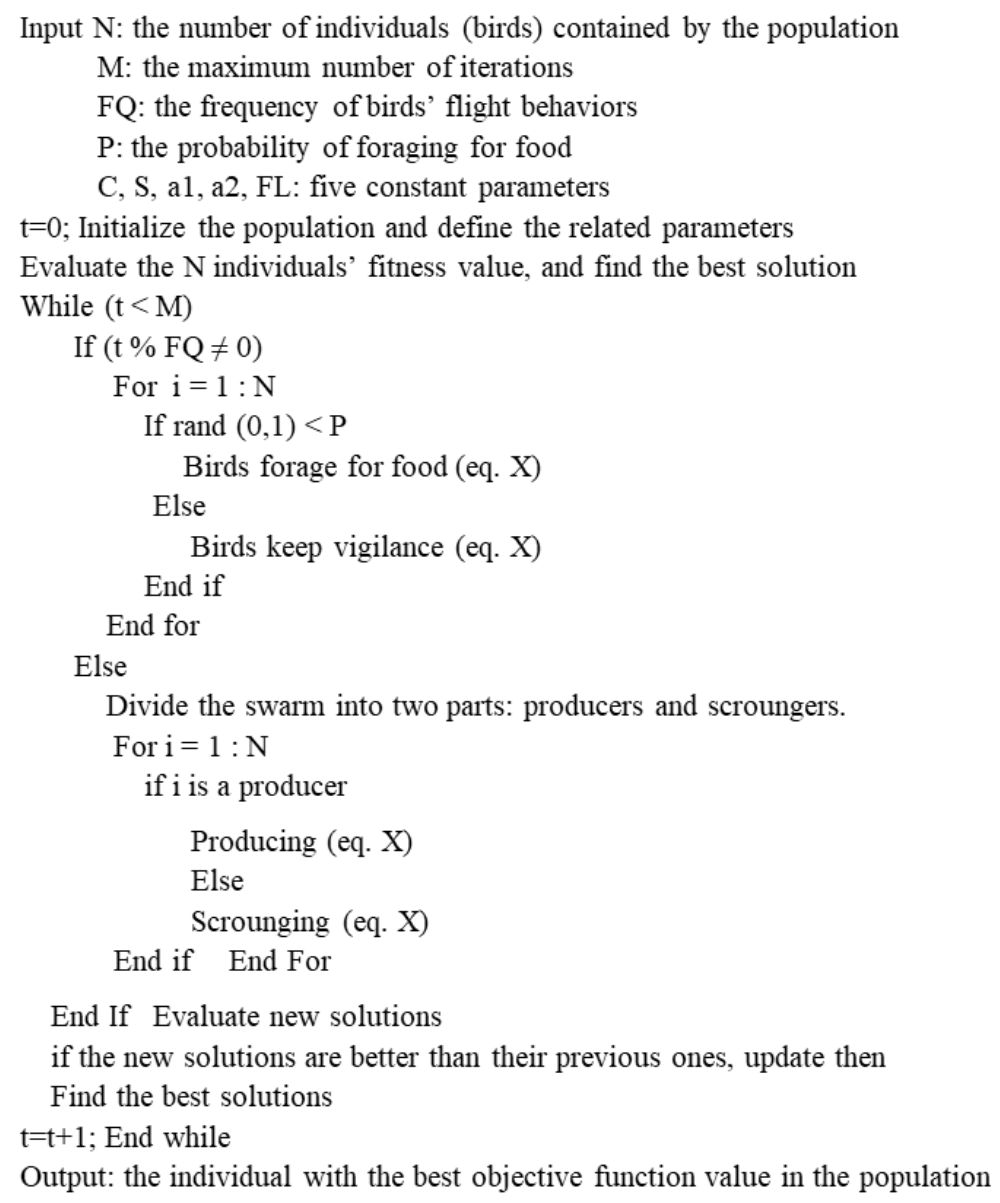

Fig 1. BSA Pseudocode

\subsection{Blood pressure}

This term is defined as the force applied against the walls of the arteries when the heart pumps blood to the body. This pressure is determined by the size and flexibility of the arteries and strength and the amount of blood pumped [19], [20]. The highest number is called Systolic pressure, which means the force exerted by the heart when pumping blood around the body. The lowest number is called Diastolic pressure and is when the heart relaxes and refills with blood, which is measured in millimeters of mercury $(\mathrm{mmHg})[19]$. 
Based on the European Guidelines for management of hypertension, the normal blood pressure should be below $139 \mathrm{mmHg}$ in systolic pressure and below $89 \mathrm{mmHg}$ in diastolic pressure [21].

\subsection{High Blood Pressure}

High blood pressure (also called hypertension) is the sustained elevation of blood

pressure above normal limits [22]. The European Guidelines for management of hypertension classifies this condition in three grades:

- Grade 1: is 140-159 mmHg in systolic pressure or 90-99 $\mathrm{mmHg}$ in diastolic pressure.

- Grade 2: is $160-179 \mathrm{mmHg}$ in systolic pressure or $100-109 \mathrm{mmHg}$ in diastolic pressure.

- Grade 3: is 180 or higher $\mathrm{mmHg}$ in systolic pressure or 110 or higher $\mathrm{mmHg}$ in diastolic pressure.

Additionally, another classification is defined, which is called Isolated Systolic Hypertension and this may occur when the Systolic pressure is higher or equal to 140 mmHg. However, the Diastolic pressure is lower than $90 \mathrm{mmHg}$ [21].

This condition does not present symptoms, so it can be confused with those of another disease. Some symptoms of severe hypertension are headaches, chest pain, nosebleeds, difficulty breathing, and dizziness [23].

The exact causes by which a person can develop hypertension are not known, but are associated with the following risk factors: physical inactivity, alcohol and tobacco use, obesity, stress, salt consumption, and age [23]. 


\subsection{Framingham Heart Study}

The Framingham Heart Study began in 1948 [24], directed by the National Cardiac Institute, which was carried out to identify risk factors that have been the cause for the development of cardiovascular diseases.

This study begins by recruiting a group of 5,209 men and women from Framingham, aged between 30 and 62 years old, who did not have any symptoms of cardiovascular disease or suffered a heart attack or stroke. Throughout the study, different groups of people have been added, such as the offspring of the first group in 1971, the multicultural group Omni in 1994, the third generation of the offspring's in 2002, and the second group of Omni in 2003 [24].

So far, different risk functions have been developed for the identification of other diseases, such as arterial fibrillation, coronary disease, diabetes, to name a few.

One of the diseases that is included in these risk functions is high blood pressure, that is, with this function, which is a Weibull regression model, we can find the percentage of risk that a patient has in developing hypertension in 4 years. To obtain the diagnosis, patient information is used, such as Age, Systolic Blood Pressure (SBP), Diastolic Blood Pressure (DBP), Body Mass Index (BMI), Sex, Smoking, Parental Hypertension.

The mathematical expression for the risk of developing hypertension is the following:

$$
\text { FHS predictor risk }=1-\exp \left[-\exp \left(\frac{\ln (4)-22.94954+\sum_{i=1}^{8} x_{i}}{0.8769}\right)\right]
$$

where:

$$
\mathrm{x}_{1}=-0.15641 * \text { Age, } \mathrm{x}_{2}=-0.20293 * \operatorname{Sex}, \mathrm{x}_{3}=-0.05933 * \mathrm{SBP} \mathrm{x}_{4}=-0.12847 * \mathrm{DBP}, \mathrm{x}_{5}=-
$$

$0.19073 *$ Smoking, $\mathrm{x}_{6}=-0.16612 *$ Parental_Hypertension, $\mathrm{x}_{7}=-0.03388 * \mathrm{BMI}, \mathrm{x}_{8}=$ $0.00162 * \mathrm{DBP}^{*}$ Age 
If the patient smokes, then the variable Smoking is assigned a value of one, otherwise it is assigned to 0 .

If none of the parents are hypertensive the variable Parental_Hypertension is assigned to 0, otherwise if one of the parents is hypertensive the it is assigned to 1 and finally, if both of the parents are hypertensive then it is assigned to 2 [24].

\section{Related works}

The fuzzy dynamic parameter adaptation has been carried out in different algorithms to solve various problems. A sample of these recent related works is presented below.

In the work of Gaxiola et al. [25], the authors proposed to perform dynamic parameter adjustment to the Particle Swarm Optimization algorithm using Interval Type-2 fuzzy systems. The main goal was to find optimal results for a particular problem, in this case for the optimization of a fuzzy neural network. The variables that are dynamically adjusted in the PSO are $c 1$ and $c 2$, which correspond to the cognitive and social factors, respectively. The proposed method was applied to the prediction of financial time series (stock exchanges in Germany, Mexico, Dow-Jones, London, Nasdaq, Shanghai, and Taiwan) and these results are compared with those obtained through a traditional neural network. Statistically, it is found that when performing the dynamic parameters adjustment to the PSO it provides better results than a non-optimized neural network.

To optimize different Benchmark control problems, Peraza et al. [26] proposed the dynamic parameter adaptation for the the Harmony Search Algorithm (HS) for optimizing the fuzzy controller of the the ball and beam system. In this case, the harmony memory accepting (HMR) parameter changes in a fuzzy way to control the exploration. Different types of fuzzy systems (type-1 fuzzy system, interval type-2 fuzzy system, and general type-2 fuzzy 
systems) were used for the proposed method. When tests of the different fuzzy types are carried out, with the bar and beam controller, it can be observed that better results are obtained by adapting parameters with the general type- 2 fuzzy system, even when levels of noise are applied to the plant.

In another work by M. Lagunes et al. [27], they proposed to perform the fuzzy dynamic parameter adjustment to the firefly algorithm (FA) using Type-1 fuzzy systems and Interval Type-2 fuzzy systems. The proposed method adjusts the alpha variable of the algorithm, which corresponds to the exploration. To test the performance of the proposed method, different fuzzy controllers are used, such as the water level control tank and the temperature control in a shower, and it is observed that the results of adjusting with the fuzzy systems are very similar. However, when adding noise, the interval type- 2 fuzzy system is better. Neural networks have been used to solve problems in medical areas [28], and in this regard, the following works can be mentioned:

In the work of Mahboob et al. [29], they used different classification algorithms for the early prediction of diabetes. Several tools were also used to determine the selection of attributes and for the grouping, prediction, and mining of association rules for the mentioned disease, finding that the body mass index and glucose level are the most important attributes associated with developing diabetes. Similarly, different classification algorithms were used to observe which of them produced better results, such as random forest, K-means clustering, and Artificial neural networks. The neural networks provide the best accuracy with $75.7 \%$. Analyzing the obtained results, it can be concluded that neural networks present better results than the other used methods, having an accuracy of $75.7 \%$. The limitation that authors face is using a structured dataset. 
In the work of R. Sadek. et al. [30], a neural network is utilized to predict Parkinson's disease since it is difficult for doctors to detect at early stages. To make this prediction they use 19 input variables, which are audio sections that were obtained from 31 people, of which 23 had Parkinson's disease. The patient's status is given as an output; this means it has a disease or is a healthy patient. As a result, precision of $100 \%$ is obtained when making the prediction.

Finally, for the recognition and classification of different types of intestinal parasites Takam et al. [31] proposed a neuro-fuzzy system, which automatically classifies these parasites by microscopic images, which are provided as input. These images are preprocessed with different techniques for the detection of parasites and once having this, the information is provided to the neuro-fuzzy classifier to perform the classification. When comparing the results contained with other authors, it can be concluded that the proposed method provides better results, $100 \%$ of recognition and classification are obtained in 20 classes of human intestinal parasites.

In other areas, neural networks and bio-inspired algorithms have been used to solve different problems, such as in the following works:

Regarding new swarm optimization methods, S. Mirjalili et al. [32] presented two new algorithms in 2017, which are called Salp Swarm Algorithm (SSA) and Multi-objective Salp Swarm Algorithm (MSSA) to solve optimization problems with single and multiple objectives, respectively. These algorithms are inspired by the behavior of the swarm of salps when navigating and feeding in the oceans. The first test performed with both algorithms deals with mathematical functions, obtaining as a result that the SSA algorithm improves the initial random solutions effectively and can converge towards the optimum, while the 
MSSA can approximate optimal Pareto solutions with high convergence and coverage. When it comes to solving engineering design problems, such as airfoil design and marine propeller design, which are challenging and computationally costly, and it is shown that the proposed algorithms can solve problems with unknown and challenging search spaces.

For Benchmark function optimization, M. Parashar et al. [33] proposed the Bird Swarm Algorithm (BSA), which is inspired by the behavior and social interaction in the swarm of birds. It was demonstrated that, with the BSA, the exploration and exploitation for optimization problems are carried out effectively and quickly. When comparing the BSA with other well-known optimization algorithms, such as GSA, PSO, RGA, among others, the results in most mathematical functions are superior and exhibit better performance. Once the comparative analysis was carried out, it was concluded that the BSA could effectively solve complex optimization problems in the real world.

We are in agreement with the authors of previous works in the literature with regards that combining fuzzy logic with optimization methods obtains better results than the corresponding original methods. In addition, the proposed method can be applied for the optimization of the different soft computing techniques in some of the previous mentioned works.

\section{Problem statement and proposed method}

The Bird Swarm Algorithm uses as its inspiration the behavior and social interaction of birds. These behaviors are: foraging, vigilance, and flight, each of them is implemented to optimize problems. This work aims at improving the BSA by updating the Cognitive (C1) and Social (C2) coefficients using fuzzy systems with Triangular and Gaussian membership functions: 
this helps the algorithm in exploitation and exploration in the search space in all the iterations. The idea is to obtain the values that give us the best results and help us to satisfactorily solve the problem. To test the presented proposal, three different Mamdani-type fuzzy systems were created. The difference in each of them is in the way the rules are ordered In Fig. 2 the flow chart with the proposal of the dynamic adaptation of parameters and the general structure of the fuzzy system are illustrate.

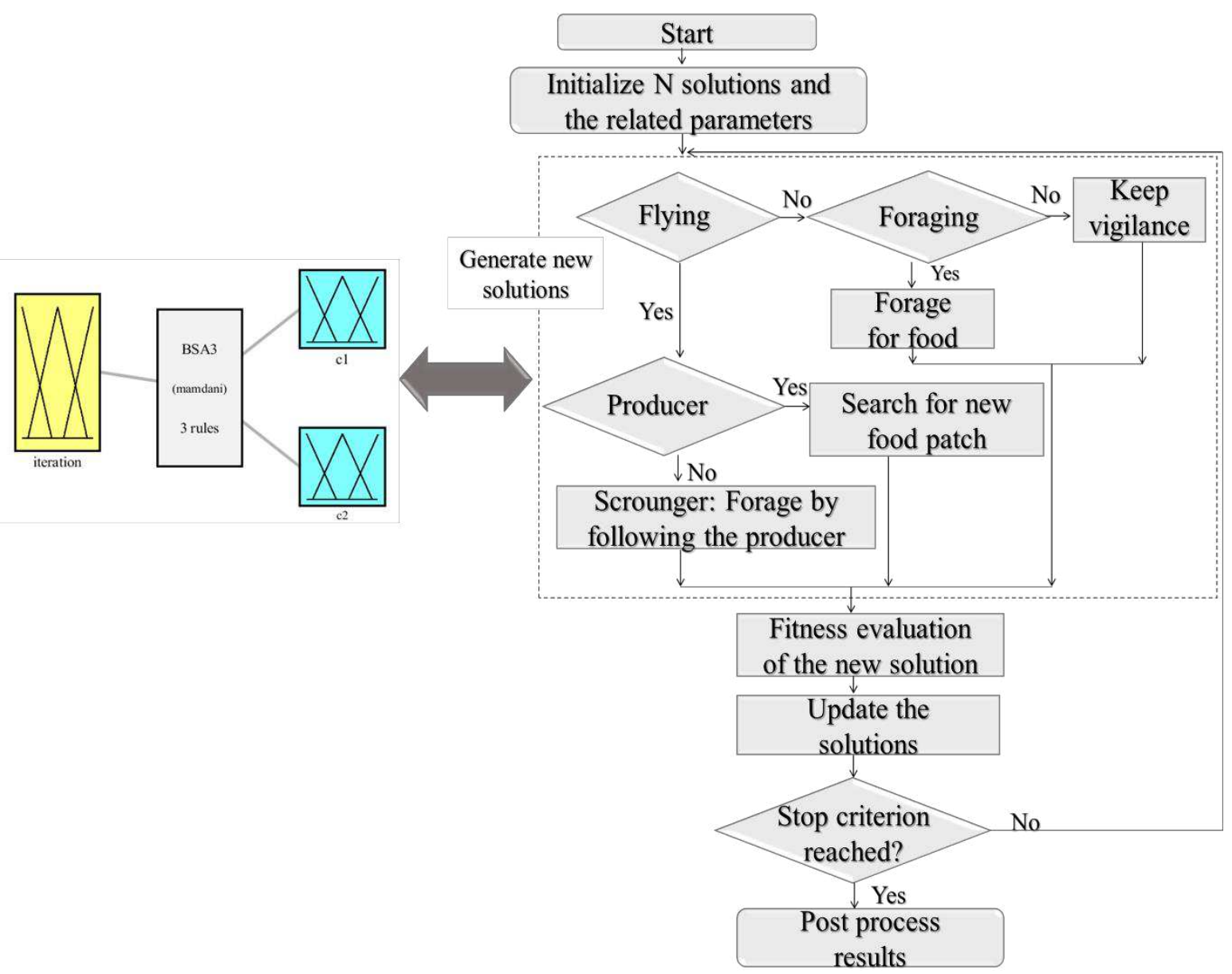

Fig 2. Proposed Fuzzy Bird Swarm method

The fuzzy system input corresponds to the iterations. To do this, the percentage of the current iteration concerning the total iterations is calculated. It means that when the algorithm 
commences, the iterations will take a low value and, in this way, it will gradually increase until reaching the point where this almost finished running the algorithm, where the iterations are going to be high or close to $100 \%$. The behavior mentioned above is summarized in the following equation [34].

$$
\text { iteration }=\frac{\text { Current iteration }}{\text { Total nummber iterations }}
$$

In Fig. 3 the input Iteration is presented, which is a granulated with 3 membership functions, using "Low", "Medium" and "High" accordingly as linguistic values.

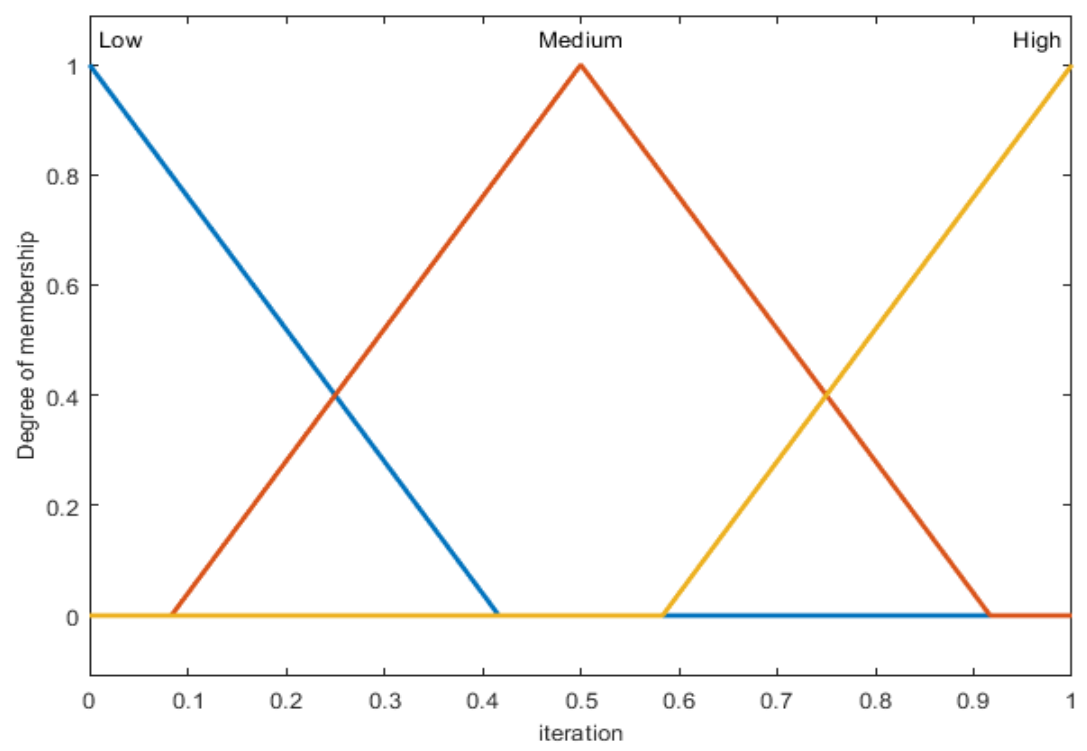

Fig 3. Input variable Iteration

The output corresponds to the parameters $\mathrm{c} 1$ and $\mathrm{c} 2$, both are granulated into three membership functions, using "Low", "Medium" and "High" as linguistic values, respectively. In Fig. 4 and Fig. 5 the fuzzy inference system outputs are presented, respectively. 


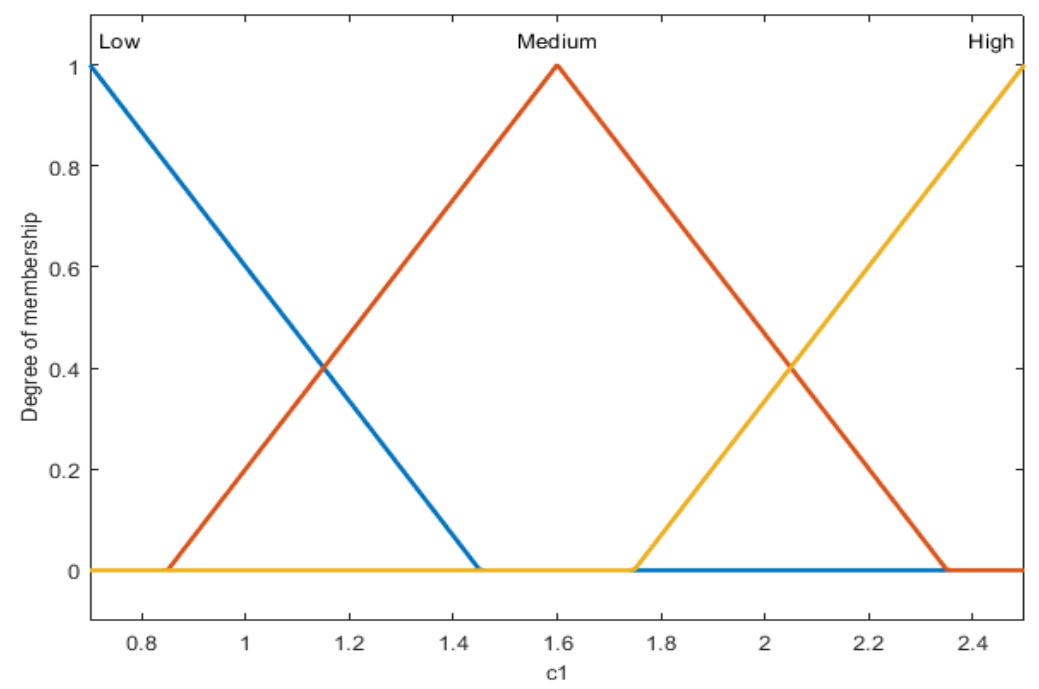

Fig 4. Output variable c1

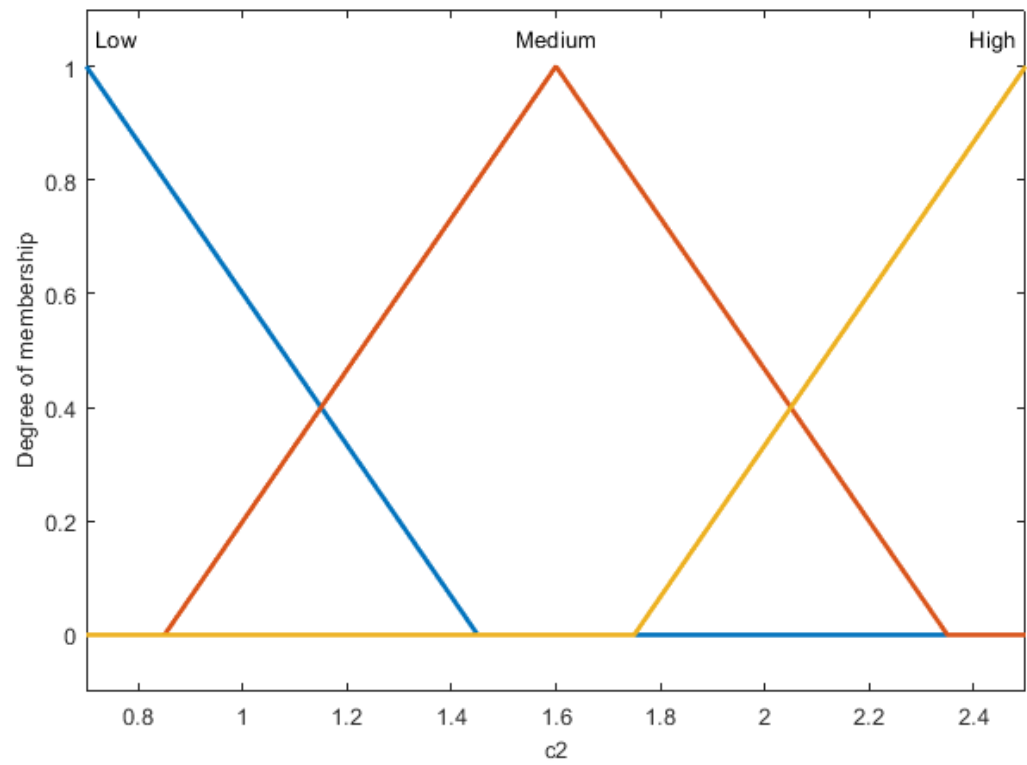

Fig 5. Output variable c2

The rules corresponding to fuzzy system number 1, where the values of c1 and c2 are increasing, are presented in Fig. 6. 


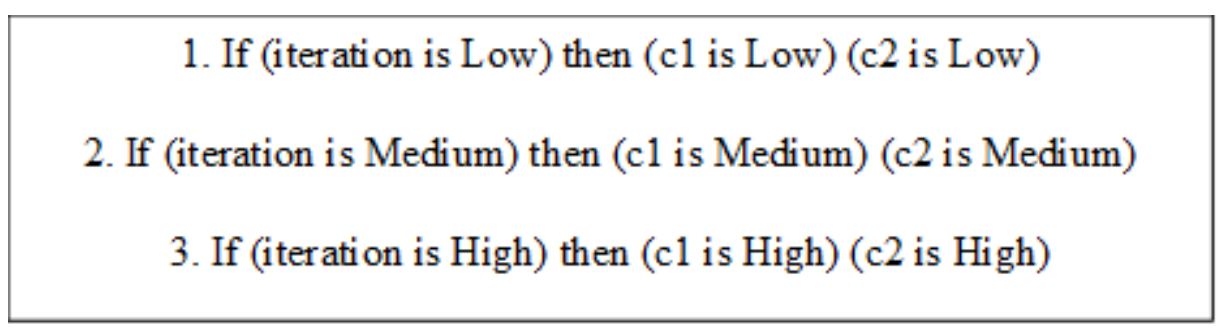

Fig 6. Rules of fuzzy system 1

In Fig. 7, the rules corresponding to fuzzy system number 2 are listed, in this case, $\mathrm{c} 1$ is increasing and $\mathrm{c} 2$ is decreasing.

1. If (iteration is Low) then ( $\mathrm{c} 1$ is Low) ( $\mathrm{c} 2$ is High)
2. If (iteration is Medium) then ( $\mathrm{c} 1$ is Medium) ( $\mathrm{c} 2$ is Medium)
3. If (iteration is High) then ( $\mathrm{c} 1$ is High) (c2 is Low)

Fig 7. Rules of fuzzy system 2

In Fig. 8, the rules corresponding to fuzzy system number 3 are listed, in this case, c1 is decreasing and $\mathrm{c} 2$ is increasing.
1. If (iteration is Low) then ( $\mathrm{c} 1$ is High) (c2 is Low)
2. If (iteration is Medium) then ( $\mathrm{c} 1$ is Medium) ( $\mathrm{c} 2$ is Medium)
3. If (iteration is High) then (c1 is Low) (c2 is High)

Fig 8 . Rules of fuzzy system 3

Taking the same parameters and the same linguistic variables, a fuzzy inference system with Gaussian membership functions was created to make a comparison and to be able to study 
which of them gives us better results. In Fig. 9 the Input is presented, while in Fig. 10 and Fig. 11 the outputs are presented, respectively.

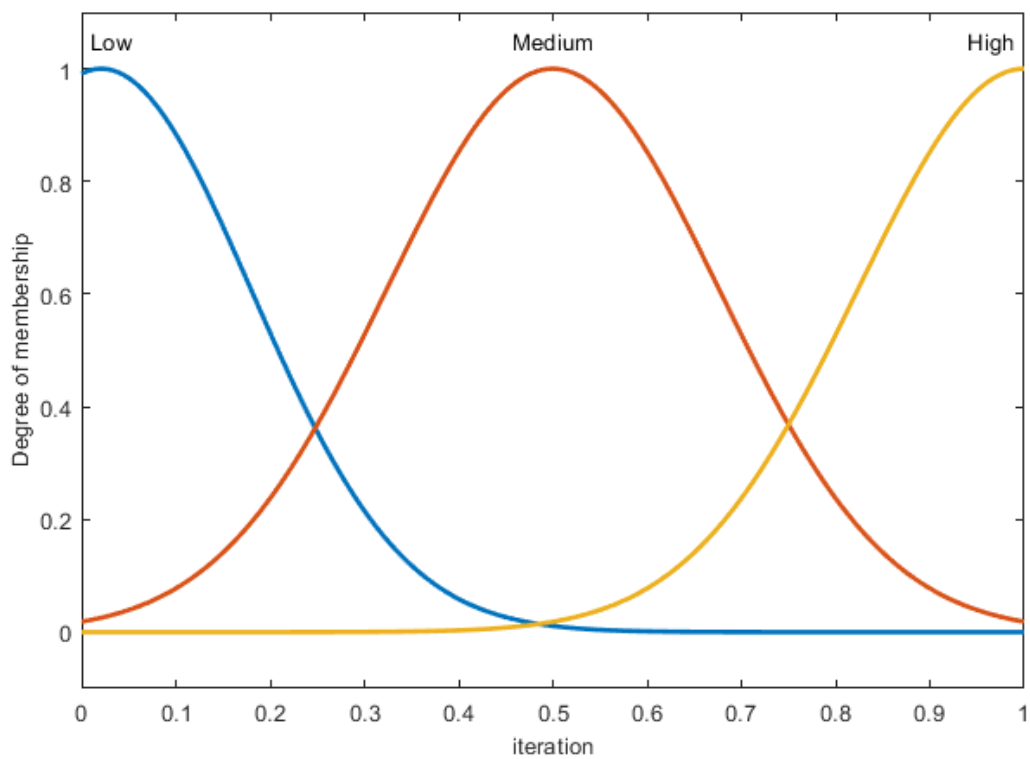

Fig 3. Input variable iteration with Gaussian membership functions

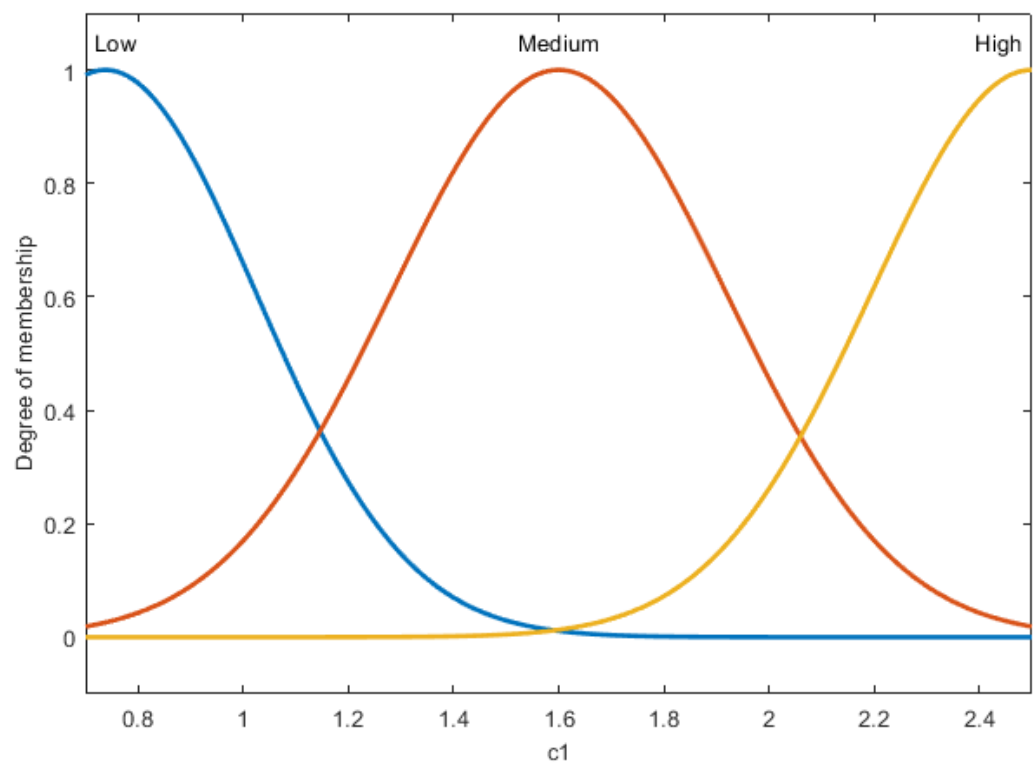

Fig 4. Output variable c1 with Gaussian membership functions 


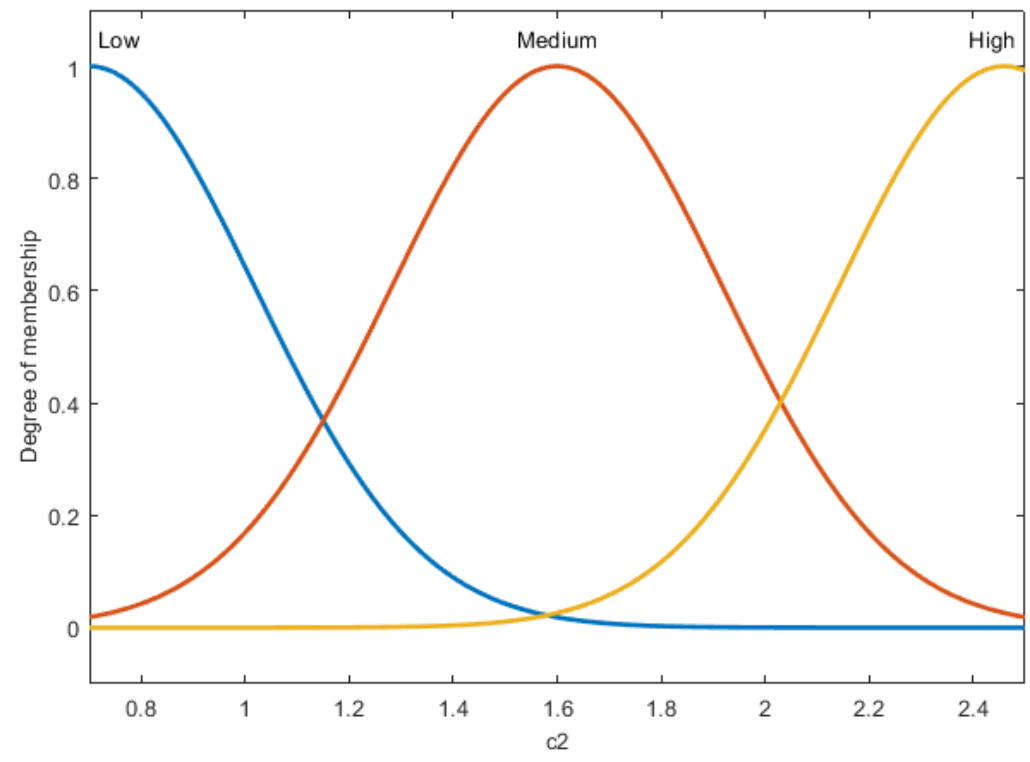

Fig 5. Output variable $\mathrm{c} 2$ with Gaussian membership functions

In the same way as in fuzzy systems with trapezoidal membership functions, the rules were varied, which are presented from Fig 6 to Fig 8.

\section{Experiments}

Different cases were considered for testing all the proposed fuzzy systems and observing which one produces the best result. For the first case, tests are performed with 10 mathematical functions ( 7 unimodal and 3 multimodal). To carry out the first set of experiments, the parameters used in the original algorithm are taken, which are presented in Table 1, where it can be observed that row 1 corresponds to the BSA and row 2 corresponds to the FBSA.

The parameters used by the algorithm, and represented in row 1 and are listed as follows:

$M$ : corresponds to the iterations 
pop: the number of individuals

dim: the dimension of the problem

$F Q$ : frequency of bird behavior

$c 1$ and $c 2$ : are the cognitive and social accelerated coefficient respectively

$a 1$ and $a 2$ : are parameters related to the indirect and direct effect of bird vigilance behavior

Table 1. Parameters used with BSA and FBSA

\begin{tabular}{|c|c|c|c|c|c|c|c|c|}
\hline & M & pop & $\operatorname{dim}$ & FQ & c1 & c2 & a1 & a2 \\
\hline $\boldsymbol{B S A}$ & 1000 & 30 & 20 & 3 & 1.5 & 1.5 & 1 & 1 \\
\hline $\boldsymbol{F B S A}$ & 1000 & 30 & 20 & 3 & Dynamic & Dynamic & 1 & 1 \\
\hline
\end{tabular}

The different mathematical functions that were used are presented in Table 2. The types of functions are presented in the first column. In the third column, the mathematical functions are presented. In columns 4 and 5, their range and their global minimum are presented, which, as we can observe, this set of functions has a value of 0 .

Table 2. Benchmark Mathematical problems

\begin{tabular}{|l|c|c|c|c|}
\hline & No & Function & Range & $f$ min \\
\hline Unimodal Benchmark & 1 & $f_{1}(x)=\sum_{1=1}^{n} x_{i}^{2}$ & {$[-100,100]$} & 0 \\
\cline { 2 - 5 } & 2 & $f_{2}(x)=\sum_{i=1}^{n}\left|x_{i}\right|+\prod_{i=1}^{n}\left|x_{i}\right|$ & {$[-10,10]$} & 0 \\
\cline { 2 - 5 } & 3 & $f_{3}(x)=\sum_{i=1}^{n}\left(\sum_{j-1}^{i} x_{j}\right)^{2}$ & {$[-100,100]$} & 0 \\
\hline
\end{tabular}




\begin{tabular}{|l|c|c|c|c|}
\hline \multirow{5}{*}{} & 4 & $f_{4}(x)=\max _{i}\left\{\left|x_{i}\right|, 1 \leq i \leq n\right\}$ & {$[-100,100]$} & 0 \\
\cline { 2 - 5 } & 5 & $f_{5}(x)=\sum_{i=1}^{n-1}\left[100\left(x_{i+1}-x_{i}^{2}\right)^{2}+\left(x_{i}-1\right)^{2}\right]$ & {$[-30,30]$} & 0 \\
\cline { 2 - 5 } & 6 & $f_{6}(x)=\sum_{i=1}^{n}\left(\left[x_{i}+0.5\right]\right)^{2}$ & {$[-100,100]$} & 0 \\
\cline { 2 - 5 } functions & 7 & $f_{7}(x)=\sum_{i=1}^{n} i x_{i}^{4}+\operatorname{random}[0,1]$ & {$[-1.28,1.28]$} & 0 \\
\cline { 2 - 5 } & 8 & $f_{9}(x)=\sum_{i=1}^{n}\left[x_{i}^{2}-10 \cos \left(2 \pi x_{i}\right)+10\right]$ & {$[-5.12,5.12]$} & 0 \\
\cline { 2 - 5 } & 9 & $f_{10}(x)=-20 \exp \left(-0.2 \sqrt{\frac{1}{n} \sum_{i=1}^{n} x_{i}^{2}}\right)$ & {$[-32,32]$} & 0 \\
\cline { 2 - 5 } & 10 & $f_{11}(x)=\frac{1}{400} \sum_{i=1}^{n} x_{i}^{2}-\prod_{i=1}^{n} \cos \left(\frac{x_{i}}{\sqrt{i}}\right)+1$ & {$[-600,600]$} & 0 \\
\hline
\end{tabular}

For the second case study, experimentation with 10 functions of the CEC2017 was performed, for this case, 50 independent runs are performed for each function and 10 experiments per function. In Table 3 the parameters used in the algorithm are presented.

Table 3. Parameters used in the second case of study

\begin{tabular}{|c|c|c|c|c|c|c|c|c|}
\hline & M & pop & $\operatorname{dim}$ & FQ & c1 & c2 & a1 & a2 \\
\hline $\boldsymbol{B S A}$ & 1500 & 30 & 30 & 3 & 1.5 & 1.5 & 1 & 1 \\
\hline $\boldsymbol{F B S A}$ & 1500 & 30 & 30 & 3 & Dynamic & Dynamic & 1 & 1 \\
\hline
\end{tabular}

The list of the different mathematical functions of CEC2017 used is presented in Table 4. In the first column, the type of functions is presented; in the second column, the number of each mathematical function is presented. The third column corresponds to the name of each function, and $F i$ corresponds to their minimum values and is presented in the fourth column. It should be noted that all these functions have a range of -100 to 100 . 
Table 4. CEC2017 benchmark functions

\begin{tabular}{|c|c|c|c|}
\hline & No & Function & $\boldsymbol{F i}$ \\
\hline \multirow{6}{*}{$\begin{array}{l}\text { Unimodal } \\
\text { Benchmark functions }\end{array}$} & 5 & Shifted and Rotated Rastrigin's Function & 500 \\
\hline & 6 & $\begin{array}{l}\text { Shifted and Rotated Expanded Scaffer's F6 } \\
\text { Function }\end{array}$ & 600 \\
\hline & 7 & $\begin{array}{l}\text { Shifted and Rotated Lunacek } \mathrm{Bi} \text { Rastrigin } \\
\text { Function }\end{array}$ & 700 \\
\hline & 8 & $\begin{array}{l}\text { Shifted and Rotated Non-Continuous Rastrigin's } \\
\text { Function }\end{array}$ & 800 \\
\hline & 9 & Shifted and Rotated Levy Function & 900 \\
\hline & 10 & Shifted and Rotated Schwefel's Function & 1000 \\
\hline Hybrid benchmark & 11 & Hybrid Function $1(\mathrm{~N}=3)$ & 1100 \\
\hline \multirow{3}{*}{$\begin{array}{l}\text { Multimodal } \\
\text { benchmark functions }\end{array}$} & 21 & Composition Function $1(\mathrm{~N}=3)$ & 2100 \\
\hline & 22 & Composition Function $2(\mathrm{~N}=3)$ & 2200 \\
\hline & 23 & Composition Function $3(\mathrm{~N}=4)$ & 2300 \\
\hline \multicolumn{4}{|c|}{$[-100,100]$} \\
\hline
\end{tabular}

To test the performance of the created fuzzy inference systems, each one was applied to a third case study, which is the optimization of a monolithic neural network. The goal of this artificial neural network was to achieve a system for obtaining the percentage of risk that a patient has in developing hypertension over four years.

This neural network is part of a neuro-fuzzy hybrid model [35]-[38] with which different results related to the patient's blood pressure are obtained; different modules used in the proposed model have been optimized [15], [39]-[42]. 
There is a database with patient information such as age, gender, body mass index, systolic and diastolic blood pressure, if the patient is a smoker, and if the patient has hypertensive parents. They are taken as inputs to the neural network. Once we have this information organized, the FBSA algorithm begins to search for the parameters of the neural network architecture, which gives us a better result. While the neural network is training the given information and learning its behavior, the FBSA changes the architecture of the neural network, in what corresponds to layers and neurons. From previous experience, and having obtained good results, it was decided that the network can have 1 to 2 hidden layers and 1 to 30 neurons per layer. In Fig.12, a representation of the optimization process is presented.

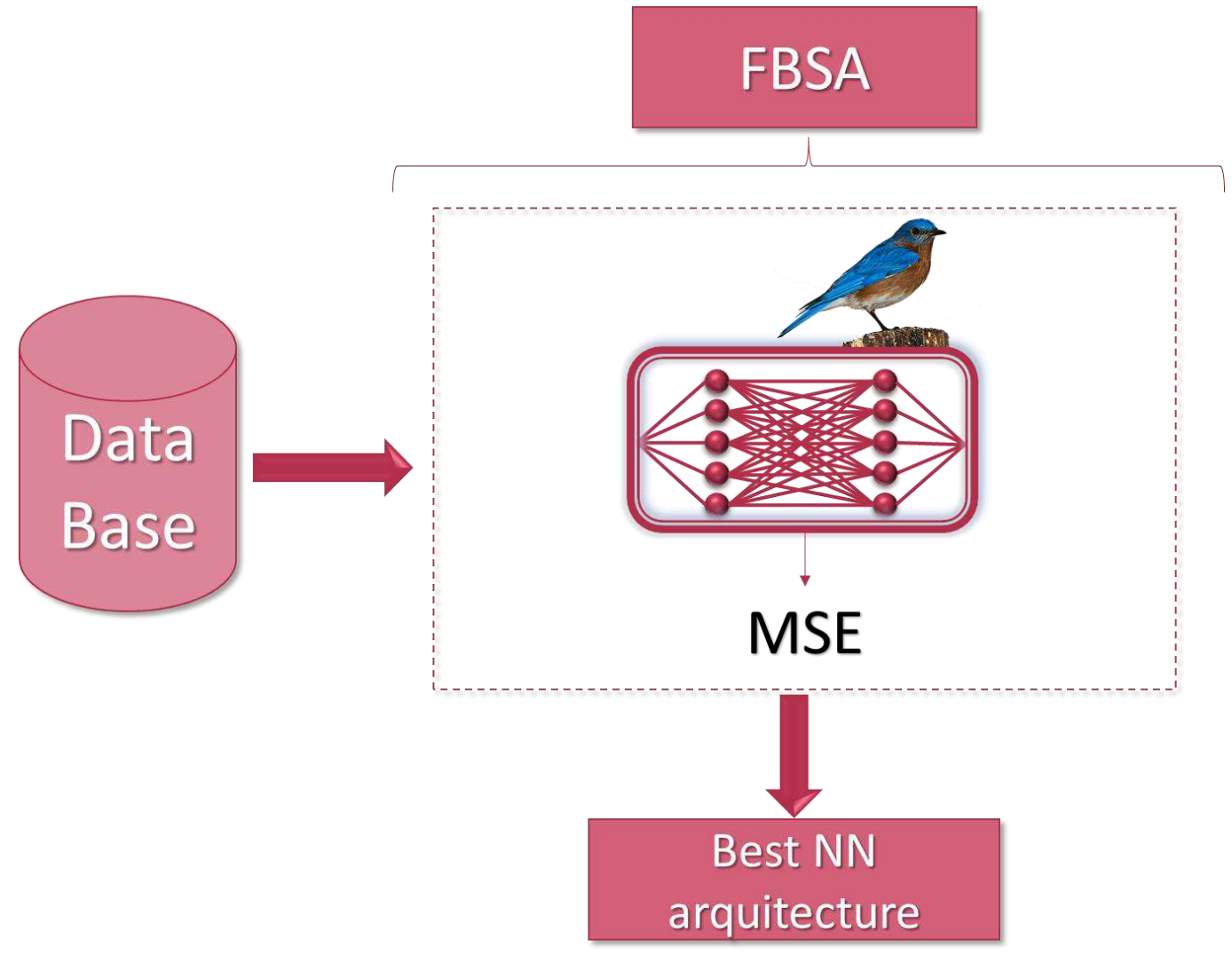

Fig 6. Neural Network Optimization 
As an objective function, the FBSA uses the mean square error (MSE), that is, the algorithm will take as the best-found architecture the one that generates the lower error, and the equation is represented as follows:

Where:

$$
M S E=\frac{1}{n} \sum_{i=1}^{n}\left(\widehat{Y}_{i}-Y_{i}\right)^{2}
$$

$\mathrm{n}=$ number of data points,

$\hat{\mathrm{Y}}=$ the value returned by the model,

$\mathrm{Y}=$ the actual value for data point $\mathrm{i}$.

\section{Results}

Table 5 presents the averages of the experiments carried out with the first set of functions. In the first column, the number of the function is indicated, in the second column the results obtained by executing the original algorithm, respecting the parameters given by the author, from the third to the eighth column the experiments carried out with the different variations of the fuzzy are presented. For these mathematical functions, an improvement of the FBSA is observed concerning the original algorithm. However, when comparing the three fuzzy inference systems, it is not observed which one is the best to improve the results of the algorithm.

Table 5. Results of the first case of study

\begin{tabular}{|c|c|c|c|c|c|c|c|}
\hline \multirow{2}{*}{ No } & \multirow{2}{*}{$\begin{array}{c}\text { Original } \\
\text { BSA }\end{array}$} & \multicolumn{2}{|c|}{ FBSA1 } & \multicolumn{2}{c|}{ FBSA2 } & \multicolumn{2}{c|}{ FBSA3 } \\
\cline { 3 - 8 } & Triangular & Gauss & Triangular & Gauss & Triangular & Gauss \\
\hline 1 & 0 & 0 & 0 & 0 & 0 & 0 & 0 \\
2 & $7.9 \mathrm{E}-212$ & $8.8 \mathrm{E}-236$ & $2.7 \mathrm{E}-241$ & $5.2 \mathrm{E}-245$ & $\mathbf{4 . 2 E}-247$ & $2 \mathrm{E}-244$ & $6 \mathrm{E}-246$ \\
3 & 0 & 0 & 0 & 0 & 0 & 0 & 0
\end{tabular}




\begin{tabular}{|c|c|c|c|c|c|c|c|}
4 & $1.4 \mathrm{E}-212$ & $8.8 \mathrm{E}-237$ & $3.4 \mathrm{E}-240$ & $4.5 \mathrm{E}-243$ & $1.1 \mathrm{E}-242$ & $8.5 \mathrm{E}-245$ & $\mathbf{1 . 7 E - 2 4 6}$ \\
5 & 18.93802 & 8.616109 & 7.920785 & $\mathbf{7 . 2 6 0 7 9}$ & 7.960495 & 7.544581 & 7.799847 \\
6 & 3.457556 & 0.057171 & 0.041854 & 0.033016 & 0.025625 & 0.022677 & $\mathbf{0 . 0 2 1 3 8 8}$ \\
7 & $7.74 \mathrm{E}-05$ & $7.38 \mathrm{E}-05$ & $7.6 \mathrm{E}-05$ & $7.6 \mathrm{E}-05$ & $\mathbf{7 . 3 E}-05$ & $7.68 \mathrm{E}-05$ & $7.52 \mathrm{E}-05$ \\
9 & 0 & 0 & 0 & 0 & 0 & 0 & 0 \\
10 & 0 & 0 & 0 & 0 & 0 & 0 & 0 \\
11 & 0 & 0 & 0 & 0 & 0 & 0 & 0 \\
\hline
\end{tabular}

The averages of the results obtained when carrying out the different experimentations are presented in Table 6. For this set of mathematical functions, the improvement for the original algorithm is noticeable, the best approach which performs better results is the fuzzy system number 3 .

Analyzing particularly the experiments carried out with the fuzzy system number 3 , it can be observed that in the fuzzy system with triangular membership functions it gives better results for 5 mathematical functions, while the fuzzy number one with Gaussian membership functions gives us good results in 3 mathematical functions. Therefore, that fuzzy 3 with triangular membership functions is the one we will use for the rest of the experimentation.

Table 6. Results of the second case of study

\begin{tabular}{|c|c|c|c|c|c|c|c|}
\hline \multirow{2}{*}{ No } & Original & \multicolumn{2}{|c|}{ FBSA1 } & \multicolumn{2}{c|}{ FBSA2 } & \multicolumn{2}{c|}{ FBSA3 } \\
\cline { 3 - 8 } & BSA & Triangular & Gauss & Triangular & Gauss & Triangular & Gauss \\
\hline 5 & $8.396 \mathrm{E}+02$ & $7.288 \mathrm{E}+02$ & $7.401 \mathrm{E}+02$ & $7.288 \mathrm{E}+02$ & $7.285 \mathrm{E}+02$ & $7.160 \mathrm{E}+02$ & $\mathbf{7 . 1 3 7 E + 0 2}$ \\
6 & $6.732 \mathrm{E}+02$ & $6.862 \mathrm{E}+02$ & $6.486 \mathrm{E}+02$ & $6.438 \mathrm{E}+02$ & $6.437 \mathrm{E}+02$ & $\mathbf{6 . 4 0 6 E}+\mathbf{0 2}$ & $6.415 \mathrm{E}+02$ \\
7 & $1.355 \mathrm{E}+03$ & $1.067 \mathrm{E}+03$ & $1.097 \mathrm{E}+03$ & $1.067 \mathrm{E}+03$ & $1.063 \mathrm{E}+03$ & $\mathbf{1 . 0 4 5 E}+\mathbf{0 3}$ & $1.049 \mathrm{E}+03$ \\
8 & $1.075 \mathrm{E}+03$ & $9.935 \mathrm{E}+02$ & $1.000 \mathrm{E}+03$ & $9.935 \mathrm{E}+02$ & $9.933 \mathrm{E}+02$ & $9.917 \mathrm{E}+02$ & $\mathbf{9 . 8 5 7 E}+\mathbf{0 2}$ \\
9 & $7.602 \mathrm{E}+03$ & $3.432 \mathrm{E}+03$ & $4.091 \mathrm{E}+03$ & $3.432 \mathrm{E}+03$ & $3.445 \mathrm{E}+03$ & $\mathbf{3 . 2 9 6 E}+\mathbf{0 3}$ & $3.337 \mathrm{E}+03$ \\
10 & $7.243 \mathrm{E}+03$ & $7.138 \mathrm{E}+03$ & $\mathbf{7 . 0 8 2 E}+\mathbf{0 3}$ & $7.138 \mathrm{E}+03$ & $7.151 \mathrm{E}+03$ & $7.190 \mathrm{E}+03$ & $7.168 \mathrm{E}+03$ \\
11 & $5.349 \mathrm{E}+03$ & $1.632 \mathrm{E}+03$ & $1.767 \mathrm{E}+03$ & $1.632 \mathrm{E}+03$ & $1.639 \mathrm{E}+03$ & $1.625 \mathrm{E}+03$ & $\mathbf{1 . 6 1 0 E}+\mathbf{0 3}$ \\
21 & $2.645 \mathrm{E}+03$ & $2.503 \mathrm{E}+03$ & $2.520 \mathrm{E}+03$ & $2.503 \mathrm{E}+03$ & $2.504 \mathrm{E}+03$ & $\mathbf{2 . 4 9 2 E + 0 3}$ & $2.500 \mathrm{E}+03$ \\
22 & $8.184 \mathrm{E}+03$ & $3.691 \mathrm{E}+03$ & $3.804 \mathrm{E}+03$ & $3.691 \mathrm{E}+03$ & $\mathbf{3 . 6 7 0 E}+\mathbf{0 3}$ & $3.831 \mathrm{E}+03$ & $3.716 \mathrm{E}+03$ \\
23 & $3.352 \mathrm{E}+03$ & $2.997 \mathrm{E}+03$ & $3.040 \mathrm{E}+03$ & $2.997 \mathrm{E}+03$ & $2.988 \mathrm{E}+03$ & $\mathbf{2 . 9 6 1 E}+\mathbf{0 3}$ & $2.991 \mathrm{E}+03$ \\
\hline
\end{tabular}


In Table 7 the parameters used in the experimentation carried out with the BSA algorithm are presented, it should be remembered that for each experiment the parameters were being varied.

Table 7. Parameters used for BSA in the third case of study

\begin{tabular}{|c|c|c|c|c|c|c|c|c|}
\hline Exp. & Iteration & Population & Frequency & $\begin{array}{c}\text { Cognitive } \\
\text { A.C } \\
\end{array}$ & $\begin{array}{c}\text { Social } \\
\text { A.C } \\
\end{array}$ & a1 & $\mathbf{a} 2$ & Dim \\
\hline 1 & 400 & 10 & 11 & 0.5 & 0.5 & 2 & 2 & 3 \\
\hline 2 & 333 & 12 & 5 & 0.8 & 0.8 & 1.5 & 1.5 & 3 \\
\hline 3 & 285 & 14 & 14 & 1.2 & 1.2 & 0.4 & 0.4 & 3 \\
\hline 4 & 250 & 16 & 11 & 1.5 & 1.5 & 0.1 & 0.1 & 3 \\
\hline 5 & 222 & 18 & 6 & 1.8 & 1.8 & 0.8 & 0.8 & 3 \\
\hline 6 & 200 & 20 & 4 & 2 & 2 & 1 & 1 & 3 \\
\hline 7 & 181 & 22 & 14 & 2.33 & 2.33 & 1.3 & 1.3 & 3 \\
\hline 8 & 166 & 24 & 15 & 2.48 & 2.48 & 0.6 & 0.6 & 3 \\
\hline 9 & 153 & 26 & 6 & 2.76 & 2.76 & 0.9 & 0.9 & 3 \\
\hline 10 & 142 & 28 & 4 & 3 & 3 & 1.1 & 1.1 & 3 \\
\hline 11 & 133 & 30 & 9 & 3.18 & 3.18 & 1.9 & 1.9 & 3 \\
\hline 12 & 125 & 32 & 14 & 3.22 & 3.22 & 0.5 & 0.5 & 3 \\
\hline 13 & 117 & 34 & 4 & 3.45 & 3.45 & 1.5 & 1.5 & 3 \\
\hline 14 & 111 & 36 & 10 & 3.56 & 3.56 & 0.7 & 0.7 & 3 \\
\hline 15 & 105 & 38 & 7 & 4 & 4 & 1.3 & 1.3 & 3 \\
\hline 16 & 100 & 40 & 10 & 0.4 & 0.4 & 1.8 & 1.8 & 3 \\
\hline 17 & 95 & 42 & 3 & 0.7 & 0.7 & 0.3 & 0.3 & 3 \\
\hline 18 & 90 & 44 & 11 & 1.15 & 1.15 & 0.9 & 0.9 & 3 \\
\hline 19 & 86 & 46 & 7 & 1.34 & 1.34 & 1 & 1 & 3 \\
\hline 20 & 83 & 48 & 3 & 1.45 & 1.45 & 2 & 2 & 3 \\
\hline 21 & 80 & 50 & 5 & 1.67 & 1.67 & 0.6 & 0.6 & 3 \\
\hline 22 & 76 & 52 & 7 & 1.78 & 1.78 & 0.3 & 0.3 & 3 \\
\hline 23 & 74 & 54 & 4 & 1.92 & 1.92 & 1.5 & 1.5 & 3 \\
\hline 24 & 71 & 56 & 5 & 2.18 & 2.18 & 1.2 & 1.2 & 3 \\
\hline 25 & 68 & 58 & 8 & 2.39 & 2.39 & 1.8 & 1.8 & 3 \\
\hline 26 & 66 & 60 & 14 & 2.56 & 2.56 & 0.7 & 0.7 & 3 \\
\hline 27 & 64 & 62 & 4 & 2.83 & 2.83 & 0.9 & 0.9 & 3 \\
\hline 28 & 62 & 64 & 8 & 3.4 & 3.4 & 1.5 & 1.5 & 3 \\
\hline 29 & 58 & 68 & 11 & 3.7 & 3.7 & 1.7 & 1.7 & 3 \\
\hline 30 & 57 & 70 & 7 & 4 & 4 & 2 & 2 & 3 \\
\hline
\end{tabular}


Table 8 presents the parameters used with the FBSA algorithm, and it should be noted here that variables c1 and c2 are dynamic with the help of the fuzzy system.

Table 8. Parameter used by FBSA in the third case study

\begin{tabular}{|c|c|c|c|c|c|c|c|c|}
\hline Exp. & Iteration & Population & Frequency & $\begin{array}{c}\text { Cognitive } \\
\text { A.C }\end{array}$ & Social A.C & a1 & a2 & Dim \\
\hline 1 & 93 & 10 & 11 & Dynamic & Dynamic & 2 & 2 & 3 \\
\hline 2 & 78 & 12 & 5 & Dynamic & Dynamic & 1.5 & 1.5 & 3 \\
\hline 3 & 66 & 14 & 14 & Dynamic & Dynamic & 0.4 & 0.4 & 3 \\
\hline 4 & 58 & 16 & 11 & Dynamic & Dynamic & 0.1 & 0.1 & 3 \\
\hline 5 & 52 & 18 & 6 & Dynamic & Dynamic & 0.8 & 0.8 & 3 \\
\hline 6 & 47 & 20 & 4 & Dynamic & Dynamic & 1 & 1 & 3 \\
\hline 7 & 42 & 22 & 14 & Dynamic & Dynamic & 1.3 & 1.3 & 3 \\
\hline 8 & 39 & 24 & 15 & Dynamic & Dynamic & 0.6 & 0.6 & 3 \\
\hline 9 & 36 & 26 & 6 & Dynamic & Dynamic & 0.9 & 0.9 & 3 \\
\hline 10 & 33 & 28 & 4 & Dynamic & Dynamic & 1.1 & 1.1 & 3 \\
\hline 11 & 31 & 30 & 9 & Dynamic & Dynamic & 1.9 & 1.9 & 3 \\
\hline 12 & 29 & 32 & 14 & Dynamic & Dynamic & 0.5 & 0.5 & 3 \\
\hline 13 & 27 & 34 & 4 & Dynamic & Dynamic & 1.5 & 1.5 & 3 \\
\hline 14 & 26 & 36 & 10 & Dynamic & Dynamic & 0.7 & 0.7 & 3 \\
\hline 15 & 24 & 38 & 7 & Dynamic & Dynamic & 1.3 & 1.3 & 3 \\
\hline 16 & 23 & 40 & 10 & Dynamic & Dynamic & 1.8 & 1.8 & 3 \\
\hline 17 & 22 & 42 & 3 & Dynamic & Dynamic & 0.3 & 0.3 & 3 \\
\hline 18 & 21 & 44 & 11 & Dynamic & Dynamic & 0.9 & 0.9 & 3 \\
\hline 19 & 20 & 46 & 7 & Dynamic & Dynamic & 1 & 1 & 3 \\
\hline 20 & 19 & 48 & 3 & Dynamic & Dynamic & 2 & 2 & 3 \\
\hline 21 & 19 & 50 & 5 & Dynamic & Dynamic & 0.6 & 0.6 & 3 \\
\hline 22 & 18 & 52 & 7 & Dynamic & Dynamic & 0.3 & 0.3 & 3 \\
\hline 23 & 17 & 54 & 4 & Dynamic & Dynamic & 1.5 & 1.5 & 3 \\
\hline 24 & 17 & 56 & 5 & Dynamic & Dynamic & 1.2 & 1.2 & 3 \\
\hline 25 & 16 & 58 & 8 & Dynamic & Dynamic & 1.8 & 1.8 & 3 \\
\hline 26 & 16 & 60 & 14 & Dynamic & Dynamic & 0.7 & 0.7 & 3 \\
\hline 27 & 15 & 62 & 4 & Dynamic & Dynamic & 0.9 & 0.9 & 3 \\
\hline 28 & 15 & 64 & 8 & Dynamic & Dynamic & 1.5 & 1.5 & 3 \\
\hline 29 & 14 & 66 & 11 & Dynamic & Dynamic & 1.7 & 1.7 & 3 \\
\hline 30 & 14 & 68 & 7 & Dynamic & Dynamic & 2 & 2 & 3 \\
\hline
\end{tabular}


In Table 9, the errors generated in each experiment are presented, and it can be observed that a minimal error is obtained when using the FBSA compared to the BSA. Regarding the used fuzzy system, it is observed that a better result is obtained with the FBSA that uses the fuzzy system number 3 for parameter adaptation.

Table 9. Errors obtained in the optimization of neural network

\begin{tabular}{|c|c|c|c|c|c|c|c|}
\hline \multirow{2}{*}{ Exp } & \multirow{2}{*}{$\begin{array}{c}\text { Original } \\
\text { BSA }\end{array}$} & \multicolumn{2}{|c|}{ FBSA1 } & \multicolumn{2}{|c|}{ FBSA2 } & \multicolumn{2}{|c|}{ FBSA3 } \\
\hline & & Tapezoidal & Gauss & Trapezoidal & Gauss & Trapezoidal & Gauss \\
\hline 1 & 7.184E-04 & $1.064 \mathrm{E}-03$ & $2.646 \mathrm{E}-04$ & 1.195E-04 & $3.363 \mathrm{E}-04$ & $1.311 \mathrm{E}-04$ & $1.183 \mathrm{E}-03$ \\
\hline 2 & $1.042 \mathrm{E}-03$ & 1.193E-03 & $3.655 \mathrm{E}-04$ & $2.784 \mathrm{E}-04$ & $2.972 E-03$ & $2.384 \mathrm{E}-04$ & 1.292E-04 \\
\hline 3 & $9.218 \mathrm{E}-04$ & $1.115 \mathrm{E}-03$ & $1.984 \mathrm{E}-04$ & $8.863 E-04$ & $1.525 \mathrm{E}-03$ & $6.635 \mathrm{E}-04$ & $1.499 \mathrm{E}-03$ \\
\hline 4 & $9.963 \mathrm{E}-04$ & 8.247E-04 & $3.663 \mathrm{E}-04$ & $8.218 \mathrm{E}-04$ & $2.037 \mathrm{E}-04$ & $5.593 \mathrm{E}-04$ & $2.624 \mathrm{E}-04$ \\
\hline 5 & $1.009 \mathrm{E}-03$ & $1.154 \mathrm{E}-03$ & $3.196 \mathrm{E}-04$ & $2.089 \mathrm{E}-04$ & 5.498E-04 & $1.927 \mathrm{E}-04$ & 4.992E-04 \\
\hline 6 & $1.107 \mathrm{E}-03$ & $4.433 E-03$ & $1.799 \mathrm{E}-04$ & $6.735 \mathrm{E}-04$ & $6.283 \mathrm{E}-04$ & $4.509 \mathrm{E}-04$ & $2.701 \mathrm{E}-04$ \\
\hline 7 & $9.542 \mathrm{E}-04$ & 8.933E-04 & $3.041 \mathrm{E}-04$ & $6.683 \mathrm{E}-04$ & $3.422 \mathrm{E}-04$ & $1.317 \mathrm{E}-04$ & $2.493 \mathrm{E}-04$ \\
\hline 8 & $9.075 \mathrm{E}-04$ & 8.824E-04 & 7.341E-04 & $1.522 \mathrm{E}-04$ & $2.485 \mathrm{E}-04$ & $4.076 \mathrm{E}-04$ & $1.865 \mathrm{E}-04$ \\
\hline 9 & 7.283E-04 & $1.225 \mathrm{E}-03$ & 4.443E-04 & $3.098 \mathrm{E}-04$ & $2.916 \mathrm{E}-04$ & $5.83 \mathrm{E}-04$ & $1.497 \mathrm{E}-04$ \\
\hline 10 & 7.961E-04 & 5.273E-04 & $7.515 E-04$ & $3.160 \mathrm{E}-04$ & $3.337 \mathrm{E}-04$ & $3.227 \mathrm{E}-04$ & $1.931 \mathrm{E}-04$ \\
\hline 11 & $1.222 \mathrm{E}-03$ & 8.012E-04 & $2.430 \mathrm{E}-04$ & $3.277 \mathrm{E}-04$ & 2.799E-04 & $3.017 \mathrm{E}-04$ & $1.998 \mathrm{E}-04$ \\
\hline 12 & $1.022 \mathrm{E}-03$ & $1.116 \mathrm{E}-03$ & $4.600 \mathrm{E}-04$ & $5.374 \mathrm{E}-04$ & 7.041E-04 & $3.062 \mathrm{E}-04$ & 4.779E-04 \\
\hline 13 & $9.936 \mathrm{E}-04$ & $1.388 \mathrm{E}-03$ & 3.679E-04 & $2.096 \mathrm{E}-04$ & $2.180 \mathrm{E}-04$ & $5.261 \mathrm{E}-04$ & $2.644 \mathrm{E}-04$ \\
\hline 14 & 8.945E-04 & $9.570 \mathrm{E}-04$ & $3.015 \mathrm{E}-04$ & $3.198 \mathrm{E}-04$ & $2.880 \mathrm{E}-04$ & $2.774 E-03$ & $1.742 E-03$ \\
\hline 15 & $1.511 \mathrm{E}-03$ & $1.028 \mathrm{E}-03$ & $3.926 \mathrm{E}-04$ & $1.734 \mathrm{E}-04$ & $2.733 \mathrm{E}-04$ & $1.786 \mathrm{E}-04$ & 2.764E-04 \\
\hline 16 & 1.659E-03 & $1.097 \mathrm{E}-03$ & $1.913 \mathrm{E}-04$ & $2.525 \mathrm{E}-04$ & $3.064 \mathrm{E}-04$ & $3.417 \mathrm{E}-04$ & $3.145 \mathrm{E}-04$ \\
\hline 17 & $1.850 E-03$ & $1.035 \mathrm{E}-03$ & 4.602E-04 & $3.383 \mathrm{E}-04$ & 1.742E-04 & $2.973 \mathrm{E}-04$ & 3.991E-04 \\
\hline 18 & 8.592E-04 & 8.704E-04 & 4.934E-04 & $6.952 \mathrm{E}-04$ & $1.856 \mathrm{E}-04$ & $1.671 \mathrm{E}-04$ & 3.904E-04 \\
\hline 19 & 6.106E-04 & 8.684E-04 & $2.656 \mathrm{E}-04$ & $3.435 \mathrm{E}-04$ & $2.558 \mathrm{E}-04$ & 4.042E-04 & $6.765 \mathrm{E}-04$ \\
\hline 20 & $6.402 \mathrm{E}-04$ & 7.279E-04 & 7.242E-04 & 4.967E-04 & $2.203 \mathrm{E}-04$ & $9.718 \mathrm{E}-04$ & 5.392E-04 \\
\hline 21 & $1.131 \mathrm{E}-03$ & $9.943 \mathrm{E}-04$ & $2.021 \mathrm{E}-04$ & $3.898 \mathrm{E}-04$ & 2.652E-04 & $4.426 \mathrm{E}-04$ & 5.414E-04 \\
\hline 22 & $1.389 \mathrm{E}-03$ & 8.342E-04 & $3.871 \mathrm{E}-04$ & $2.31 \mathrm{E}-04$ & 2.733E-04 & 1.134E-04 & 9.507E-04 \\
\hline 23 & $1.487 \mathrm{E}-03$ & $1.051 \mathrm{E}-03$ & $3.275 \mathrm{E}-04$ & $2.152 \mathrm{E}-04$ & $3.517 \mathrm{E}-04$ & 4.835E-04 & 4.908E-04 \\
\hline 24 & $1.185 \mathrm{E}-03$ & $1.507 \mathrm{E}-03$ & $5.577 \mathrm{E}-04$ & $3.783 \mathrm{E}-04$ & $7.105 \mathrm{E}-04$ & $1.969 \mathrm{E}-04$ & 5.511E-04 \\
\hline 25 & $1.195 \mathrm{E}-03$ & $1.435 \mathrm{E}-03$ & $3.009 \mathrm{E}-04$ & $1.714 \mathrm{E}-04$ & $5.942 \mathrm{E}-04$ & $9.670 \mathrm{E}-04$ & $2.028 \mathrm{E}-04$ \\
\hline 26 & $7.542 \mathrm{E}-04$ & $1.527 \mathrm{E}-03$ & $5.216 \mathrm{E}-04$ & $2.709 \mathrm{E}-04$ & $2.436 \mathrm{E}-04$ & $2.951 \mathrm{E}-04$ & $3.395 \mathrm{E}-04$ \\
\hline 27 & 9.913E-04 & $1.149 \mathrm{E}-03$ & 5.703E-04 & $6.130 \mathrm{E}-04$ & $5.047 \mathrm{E}-04$ & $2.113 \mathrm{E}-04$ & $1.304 \mathrm{E}-04$ \\
\hline 28 & $1.202 \mathrm{E}-03$ & 7.364E-04 & 2.682E-04 & $2.418 \mathrm{E}-04$ & $3.162 \mathrm{E}-04$ & $3.310 \mathrm{E}-04$ & $3.283 \mathrm{E}-04$ \\
\hline 29 & $1.294 \mathrm{E}-03$ & $9.611 \mathrm{E}-04$ & 7.045E-04 & $5.696 \mathrm{E}-04$ & 1.631E-04 & $1.738 \mathrm{E}-04$ & $3.782 \mathrm{E}-04$ \\
\hline 30 & $1.441 \mathrm{E}-03$ & $9.273 \mathrm{E}-04$ & $1.697 \mathrm{E}-04$ & 4.633E-04 & $3.128 \mathrm{E}-04$ & $2.563 \mathrm{E}-04$ & $9.060 \mathrm{E}-04$ \\
\hline
\end{tabular}


On average, the fuzzy system with the least error value was number two with trapezoidal membership functions. In Table 10 the averages of all experiments are presented.

Table 10. Average of the neural network optimization

\begin{tabular}{|c|c|c|c|c|c|c|}
\hline \multirow{2}{*}{ BSA } & \multicolumn{2}{|c|}{ FBSA1 } & \multicolumn{2}{c|}{ FBSA2 } & \multicolumn{2}{c|}{ FBSA3 } \\
\cline { 2 - 7 } & Trapezoidal & Gaussian & Trapezoidal & Gaussian & Trapezoidal & Gaussian \\
\hline 1.084E-03 & $1.144 \mathrm{E}-03$ & $3.946 \mathrm{E}-04$ & $\mathbf{3 . 8 9 1 E - 0 4}$ & $4.691 \mathrm{E}-04$ & $4.474 \mathrm{E}-04$ & $4.907 \mathrm{E}-04$ \\
\hline
\end{tabular}

Once having obtained the best architecture, the simulation is carried out with a group of 20 real patients. Table 11 presents the different risk factors of each patient and where the comparison of the real results of the study is made, with the ones obtained by BSA and FBSA, and it can be observed that we are having better results with the FBSA.

Table 11. Simulation of real patients

\begin{tabular}{ccccccccccc}
\hline No & Age & Gen. & BMI & Syst. & Dias. & Smoke & H.par & Real & FBSA & BSA \\
\hline 1 & 27 & M & 24.30 & 122 & 77 & No & 0 & 4 & 4 & 4 \\
2 & 28 & M & 23.36 & 120 & 81 & No & 0 & 5 & 5 & 5 \\
3 & 28 & F & 29.76 & 123 & 82 & No & 2 & 16 & 16 & 18 \\
4 & 25 & M & 24.40 & 114 & 65 & No & 0 & 1 & 1 & 1 \\
5 & 45 & M & 24.90 & 116 & 75 & No & 1 & 6 & 6 & 6 \\
6 & 31 & F & 35.26 & 95 & 61 & No & 1 & 0 & 0 & 0 \\
7 & 33 & M & 25.26 & 130 & 74 & No & 2 & 10 & 10 & 11 \\
8 & 32 & M & 29.98 & 123 & 76 & No & 1 & 7 & 7 & 8 \\
9 & 25 & M & 21.70 & 108 & 66 & No & 0 & 0 & 0 & 1 \\
10 & 30 & M & 30.30 & 123 & 78 & No & 0 & 7 & 7 & 7 \\
11 & 30 & F & 21.55 & 107 & 61 & No & 1 & 1 & 1 & 1 \\
12 & 32 & M & 24.49 & 112 & 72 & No & 0 & 2 & 2 & 2 \\
13 & 31 & F & 30.07 & 112 & 71 & No & 2 & 3 & 3 & 3 \\
14 & 29 & F & 21.50 & 99 & 62 & No & 0 & 0 & 0 & 1 \\
15 & 31 & F & 23.40 & 106 & 65 & No & 0 & 1 & 1 & 2 \\
16 & 26 & F & 31.90 & 126 & 68 & No & 0 & 4 & 4 & 4 \\
17 & 32 & M & 31.10 & 110 & 68 & No & 0 & 1 & 1 & 1 \\
18 & 30 & M & 28.91 & 122 & 76 & No & 0 & 5 & 5 & 5 \\
19 & 31 & F & 29.00 & 114 & 66 & No & 2 & 2 & 2 & 3 \\
20 & 27 & M & 22.72 & 115 & 72 & No & 1 & 2 & 2 & 2 \\
\hline & & & & & & & & & &
\end{tabular}


The best neural network architecture that was obtained is defined as:

Number of hidden layers: 2

Neurons in hidden layer 1: 8

Neurons in hidden layer 1: 6

\section{Statistical test}

To compare and verify which of the different methods yields the lowest error, a statistical test is performed, using the $\mathrm{Z}$ test, which uses the following expression:

$$
Z=\frac{\left(\bar{x}_{1}-\bar{x}_{2}\right)-\left(\mu_{1}-\mu_{2}\right)}{\sigma_{\bar{x}_{1}-\bar{x}_{2}}}
$$

where $\bar{x}_{1}-\bar{x}_{2}$ is the observed difference, $\mu_{1}-\mu_{2}$ is the expected difference and $\sigma_{\bar{x}_{1}-\bar{x}_{2}}$ is the standard error of the differences.

In the experiments of the CEC2017 functions, the null hypothesis states that the results obtained by the FBSA algorithm are greater than or equal to the results obtained by the BSA algorithm. The alternative hypothesis states that the results obtained by the FBSA algorithm are less than the results obtained by the BSA algorithm. Table 12 presents the Statistical Parameters for this problem.

Table 12. Statistical Parameters for FBSA vs BSA

\begin{tabular}{cc}
\hline Z test parameters FBSA vs BSA \\
\hline Critical Value $\left(\mathrm{Z}_{\mathrm{c}}\right)$ & -1.64 \\
Significance Level $(\alpha)$ & 0.05 \\
$\mathrm{H}_{0}$ & $\mu 1 \geq \mu 2$ \\
$\mathrm{H}_{\mathrm{a}}($ Claim $)$ & $\mu 1<\mu 2$ \\
Level of significance & $95 \%$ \\
\hline
\end{tabular}


In Table 13, the results of the $\mathrm{Z}$ test of all the functions are presented, in the first four columns, the average and standard deviation of the experiments performed with BSA and FBSA are presented respectively. In column five, the $\mathrm{Z}$ value is presented, and in the last column, the evidence is presented. In this case, "S" means the test has significant evidence and "NS" means that the test has no significance. It can be noted that in 9 of the 10 functions the evidence from the statistical test is significant, which means that there is sufficient evidence to support the alternative hypothesis, which is that the results of the FBSA are better than the BSA.

Table 13. Statistical test results for CEC2017 functions

\begin{tabular}{ccccccc}
\hline \multirow{2}{*}{ No } & \multicolumn{2}{c}{ Original } & \multicolumn{2}{c}{ FBSA } & \multirow{2}{*}{ Z value } & Evidence \\
\cline { 2 - 5 } & Average & S.D & Average & S.D & & \\
\hline 5 & 839.55 & 4.30 & 712.74 & 7.44 & -80.83 & $\mathrm{~S}$ \\
6 & 673.23 & 1.19 & 641.39 & 1.26 & -100.75 & $\mathrm{~S}$ \\
7 & 1354.97 & 8.70 & 1044.43 & 8.41 & -140.57 & $\mathrm{~S}$ \\
8 & 1075.02 & 6.52 & 986.26 & 3.16 & -67.10 & $\mathrm{~S}$ \\
9 & 7601.72 & 217.87 & 3300.65 & 105.02 & -97.40 & $\mathrm{~S}$ \\
10 & 7242.97 & 78.28 & 7240.19 & 119.18 & -0.11 & $\mathrm{NS}$ \\
11 & 5348.57 & 335.48 & 1702.34 & 250.48 & -47.70 & $\mathrm{~S}$ \\
21 & 2644.52 & 8.46 & 2486.35 & 4.51 & -90.37 & $\mathrm{~S}$ \\
22 & 8183.92 & 96.92 & 3922.18 & 261.25 & -83.77 & $\mathrm{~S}$ \\
23 & 3352.21 & 13.05 & 2980.71 & 13.70 & -107.54 & $\mathrm{~S}$ \\
\hline
\end{tabular}

In the neural network experiments, the null hypothesis expresses that the errors obtained by the FBSA algorithm are greater than or equal to the errors obtained by the BSA algorithm. The alternative hypothesis expresses that the errors obtained by the FBSA algorithm are less than the errors obtained by the BSA algorithm. Table 14 illustrates a comparison of means between the two methods with 30 experiments; it can be observed that the FBSA obtained a lower error compared with the BSA. 
Table 14. Comparison results between methods

\begin{tabular}{ccc}
\hline MSE & FBSA & BSA \\
\hline Average & $3.89 \mathrm{E}-04$ & $1.08 \mathrm{E}-03$ \\
Standard Deviation & $2.07 \mathrm{E}-04$ & $3.02 \mathrm{E}-04$ \\
Experiments & 30 & 30 \\
\hline
\end{tabular}

Table 15 presents the statistical parameters for this test.

Table 15. Statistical Parameters for FBSA vs BSA

\begin{tabular}{cc}
\hline Z test parameters FBSA vs BSA \\
\hline Test Statistic Value & -10.367 \\
Critical Value $\left(\mathrm{Z}_{\mathrm{c}}\right)$ & -1.64 \\
Significance Level $(\alpha)$ & 0.05 \\
$\mathrm{H}_{0}$ & $\mu 1 \geq \mu 2$ \\
$\mathrm{H}_{\mathrm{a}}($ Claim $)$ & $\mu 1<\mu 2$ \\
Experiments & 30 \\
\hline
\end{tabular}

Since it is observed that $\mathrm{z}$ test statistic value $\mathrm{z}=-10.367$ is less than the critical value $Z_{c}$ $=-1.64$, it is then concluded that the null hypothesis is rejected and the alternative hypothesis is accepted. It can be concluded that there is sufficient evidence with a 5\% level of significance to support the claim that the errors obtained by the FBSA algorithm are lower than the errors obtained by the BSA algorithm. In Fig. 13 the probability distribution graph for this test is illustrated. 


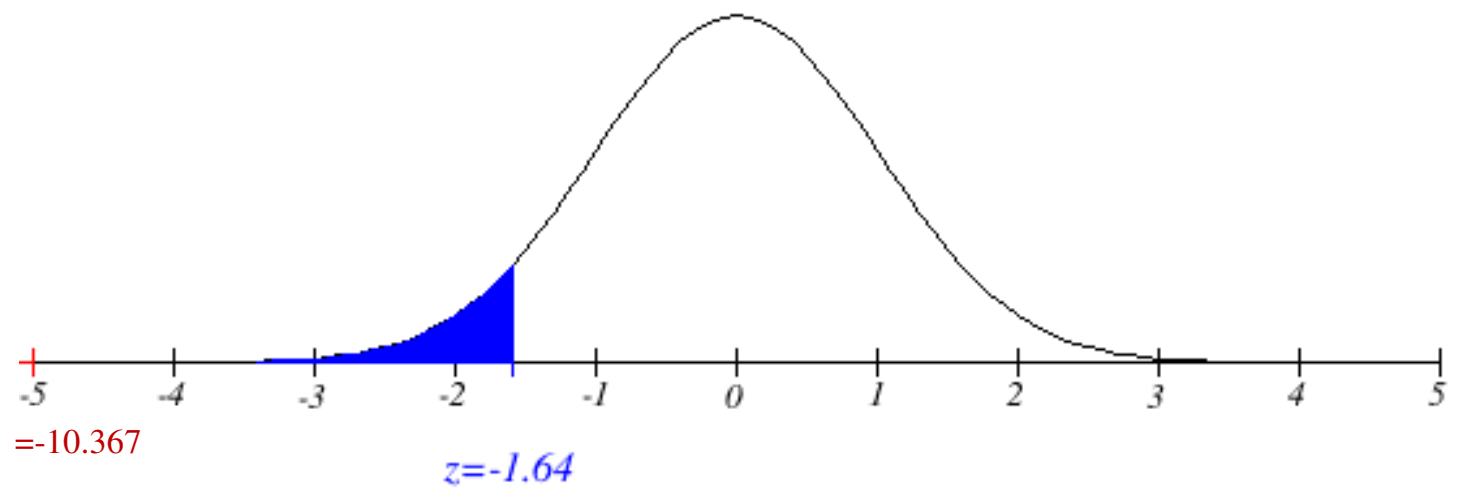

Fig 7. The plot of the Normal Distribution

\subsection{Discussion of results}

In this work, the dynamic parameter adaptation to the algorithm called FBSA was applied to mathematical functions and is utilized in a real problem: obtaining the percentage of developing hypertension in the next four years by optimizing a neural network. The objective of making the mentioned adaptation is to improve the performance of BSA to obtain better results, where it is possible to observe a significant improvement compared to the original algorithm. The results can be observed in Tables 5 and 6 for the mathematical functions and Table 9 for the case of optimization of the neural network architecture. In the case of the neural network, this is with the best architecture provided by the FBSA, and a group of patients is simulated to observe the provided results. When these are compared with the data obtained by the BSA, it is observed that the information provided by the FBSA has higher accuracy since this provides $100 \%$ of correct results; this can be verified in Table 11.

The statistical analysis demonstrates that the proposed method is significantly better in 9 of the 10 complex CEC2017 benchmark functions. Similarly, the FBSA optimization of the neural network also obtains sufficient evidence to support that it is better than BSA. 
Another experimentation that can be done in the future with the FBSA is to apply it to control problems, in addition to using Interval Type-2 Fuzzy Systems for the parameter adaptation.

\section{Conclusion}

The dynamic adaptation of parameters of the BSA algorithm is presented in this work, and this was proposed in order to improve the performance of the algorithm. Three different fuzzy systems were designed to carry out the mentioned adaptation, and the difference among the fuzzy systems was in the rules. After analysis, it was decided to make dynamic the parameters corresponding to the cognitive and social acceleration coefficients, respectively $(c 1, c 2)$. Because when varying these parameters, the variation in the results is observed.

Three different case studies were considered to determine if a better result is obtained than the original algorithm and to find out which of the 3 fuzzy systems achieves the best result. In the first case study, which was to experiment with 10 traditional mathematical functions, it can be observed that, there was an improvement in the results with the FBSA algorithm against the BSA. However, in this case a decision could not be made in which of the applied fuzzy systems was better, since the observed results were similar.

In case study number two, which was tested with 10 CEC 2017 functions, it was also observed that the FBSA provides better results. In this case, it can be concluded that for the solution of this problem the fuzzy system number 3, which uses decreasing rules and triangular membership functions provides the best result. In the last case study, which consisted of optimizing a neural network architecture to obtain the risk of developing hypertension over four years, a considerable improvement was also noted. When the methods were statistically tested, it can be observed that there is significant evidence to be able to 
affirm that the FBSA algorithm gives us better results. We can conclude that when performing dynamic adaptation of parameters to the BSA algorithm, a significant improvement in the results is obtained. In future work, we plan in applying the dynamic adaptation of parameters with Interval Type-2 Fuzzy System.

\section{Acknowledgments}

We would like to express our gratitude to the Consejo Nacional de Ciencia y Tecnologia and Tecnologico Nacional de Mexico/Tijuana Institute of Technology for the facilities and resources granted for the development of this research.

\section{Compliance with Ethical Standards:}

Funding: This research work did not receive funding

Conflict of Interest: All the authors in the paper have no conflict of interest

Ethical approval: This article does not contain any studies with human participants or animals performed by any of the authors

\section{References}

[1] W. Deng, H. Zhao, L. Zou, G. Li, X. Yang, and D. Wu, "A novel collaborative optimization algorithm in solving complex optimization problems," Soft Computing, vol. 21, no. 15, pp. 4387-4398, 2017. 
[2] S. A. Bagloee, M. Asadi, M. Sarvi, and M. Patriksson, "A hybrid machine-learning and optimization method to solve bi-level problems," Expert Systems with Applications, vol. 95, pp. 142-152, 2018.

[3] M. Ahmadigorji, N. Amjady, and S. Dehghan, "A novel two-stage evolutionary optimization method for multiyear expansion planning of distribution systems in presence of distributed generation," Applied Soft Computing, vol. 52, pp. 1098-1115, 2017.

[4] H. Badem, A. Basturk, A. Caliskan, and M. E. Yuksel, "A new hybrid optimization method combining artificial bee colony and limited-memory BFGS algorithms for efficient numerical optimization," Applied Soft Computing, vol. 70, pp. 826-844, 2018.

[5] M. Nazari-Heris and B. Mohammadi-Ivatloo, "Application of Robust Optimization Method to Power System Problems," Classical and Recent Aspects of Power System Optimization, Eds. Academic Press, pp. 19-32, 2018.

[6] X. Chang, C. Yang, and J. Xiong, "Quantized Fuzzy Output Feedback Control for Nonlinear Systems With Adjustment of Dynamic Parameters," IEEE Transactions on Systems, Man, and Cybernetics: Systems, vol. 49, no. 10, pp. 2005-2015, 2019.

[7] G. Sun, Y. Lan, and R. Zhao, "Differential evolution with Gaussian mutation and dynamic parameter adjustment," Soft Computing, vol. 23, no. 5, pp. 1615-1642, 2019.

[8] S.-F. Li and C.-Y. Cheng, "Particle swarm optimization with fitness adjustment parameters," Computers \& Industrial Engineering, vol. 113, pp. 831-841, 2017.

[9] O. Aoun, M. Sarhani, and A. El Afia, "Hidden Markov Model Classifier for the 
Adaptive Particle Swarm Optimization," Recent Developments in Metaheuristics, Eds. Cham: Springer International Publishing, 2018.

[10] Z. Assaghir, A. Janbain, S. Makki, M. Kurdi, and R. Karam, "Using Neural Network to predict the Hypertension," International Journal of Scientific Development and Research, vol. 2, no. 2, pp. 35-38, 2017.

[11] F. Olivas, F. Valdez, O. Castillo, and P. Melin, "Dynamic parameter adaptation in particle swarm optimization using interval type-2 fuzzy logic.," Soft Computing, vol. 20, no. 3, pp. 1057-1070, 2016.

[12] F. Valdez and C. Peraza, "Dynamic parameter adaptation in the harmony search algorithm for the optimization of interval type-2 fuzzy logic controllers.," Soft Computing, vol. 24, no. 1, pp. 179-192, 2020.

[13] P. Ochoa, O. Castillo, and J. Soria, "Optimization of fuzzy controller design using a Differential Evolution algorithm with dynamic parameter adaptation based on Type-1 and Interval Type-2 fuzzy systems.," Soft Computing, vol. 24, no. 1, pp. 193-214, 2020.

[14] E. Bernal, O. Castillo, J. Soria, and F. Valdez, "Fuzzy Galactic Swarm Optimization with Dynamic Adjustment of Parameters Based on Fuzzy Logic.," SN Computer Scieence, vol. 1, no. 1, p. 59, 2020.

[15] I. Miramontes, C. J. Guzman, P. Melin, and G. Prado-Arechiga, "Optimal Design of Interval Type-2 Fuzzy Heart Rate Level Classification Systems Using the Bird Swarm Algorithm," Algorithms, vol. 11, no. 12. pp. 1-35 2018.

[16] X.-B. Meng, X. Z. Gao, L. Lu, Y. Liu, and H. Zhang, “A new bio-inspired optimisation 
algorithm: Bird Swarm Algorithm," Journal of Experimental \& Theoretical Artificial Intelligence, vol. 28, no. 4, pp. 673-687, 2016.

[17] D. Maharana, R. Kommadath, and P. Kotecha, "Dynamic Yin-Yang Pair Optimization and its performance on single objective real parameter problems of CEC 2017,” 2017 IEEE Congress on Evolutionary Computation, CEC 2017 - Proceedings, pp. 23902396, 2017.

[18] R. Salgotra, U. Singh, and S. Saha, "Improved Cuckoo Search with Better Search Capabilities for Solving CEC2017 Benchmark Problems,” 2018 IEEE Congress on Evolutionary Computation, CEC 2018 - Proceedings, pp. 1-7, 2018.

[19] V. Papademetriou, E. A. Andreadis, and C. Geladari, Management of Hypertension. Cham: Springer International Publishing AG, 2019.

[20] M. Paul et al., "Measurement of Blood Pressure in Humans: A Scientific Statement From the American Heart Association," Hypertension, vol. 73, no. 5, pp. e35-e66, 2019.

[21] A. Zanchetti et al., "2018 ESC/ESH Guidelines for the management of arterial hypertension," European Heart Journal, vol. 39, no. 33, pp. 3021-3104, 2018.

[22] G. L. Bakris and M. J. Sorrentino, Hypertension, A Companion to Braunwald's Heart Disease, Third Edit. Philadelphia: Elsevier, 2018.

[23] “Texas Heart Institute,” High Blood Pressure (Hypertension), 2017. https://www.texasheart.org/heart-health/heart-information-center/topics/high-bloodpressure-hypertension/ (accessed Feb. 17, 2020). 
[24] “Framingham Heart Study," 2019. https://www.framinghamheartstudy.org/riskfunctions/hypertension/index.php (accessed Dec. 03, 2020).

[25] F. Gaxiola, P. Melin, F. Valdez, J. R. Castro, and A. Manzo-Martínez, "PSO with dynamic adaptation of parameters for optimization in neural networks with interval type-2 fuzzy numbers weights," Axioms, vol. 8, no. 1, 2019.

[26] C. Peraza, F. Valdez, J. R. Castro, and O. Castillo, "Fuzzy Dynamic Parameter Adaptation in the Harmony Search Algorithm for the Optimization of the Ball and Beam Controller," Advances in Operations Research, vol. 2018, p. 3092872, 2018.

[27] M. L. Lagunes, O. Castillo, F. Valdez, and J. Soria, "Comparison of Fuzzy Controller Optimization with Dynamic Parameter Adjustment Based on of Type-1 and Type-2 Fuzzy Logic," Hybrid Intelligent Systems in Control, Pattern Recognition and Medicine, Eds. Cham: Springer International Publishing, 2020.

[28] A. Albu, R.-E. Precup, and T.-A. Teban, "Results and challenges of artificial neural networks used for decision-making and control in medical applications," Facta universitatis, pp. 1-24, 2019.

[29] T. Mahboob Alam et al., "A model for early prediction of diabetes," Informatics in Medicine Unlocked, vol. 16, no. July, p. 100204, 2019.

[30] R. M. Sadek et al., "Parkinson's Disease Prediction Using Artificial Neural Network," International Journal of Academic Health and Medical Research (IJAHMR), vol. 3, no. 1, pp. 1-8, 2019.

[31] O. T. Nkamgang, D. Tchiotsop, B. S. Tchinda, and H. B. Fotsin, "A neuro-fuzzy system for automated detection and classification of human intestinal parasites," 
Informatics in Medicine Unlocked, vol. 13, no. October, pp. 81-91, 2018.

[32] S. Mirjalili, A. H. Gandomi, S. Z. Mirjalili, S. Saremi, H. Faris, and S. M. Mirjalili, “Salp Swarm Algorithm: A bio-inspired optimizer for engineering design problems," Advances in Engineering Software, vol. 114, pp. 163-191, 2017.

[33] M. Parashar, S. Rajput, H. M. Dubey, and M. Pandit, “Optimization of benchmark functions using a nature inspired bird swarm algorithm," 2017 3rd International Conference on Computational Intelligence \& Communication Technology (CICT), pp. $1-7,2017$.

[34] P. Melin, F. Olivas, O. Castillo, F. Valdez, J. Soria, and J. M. G. Valdez, “Optimal design of fuzzy classification systems using PSO with dynamic parameter adaptation through fuzzy logic.," Expert Systems whit Applications., vol. 40, no. 8, pp. 3196$3206,2013$.

[35] P. Melin, I. Miramontes, and G. Prado-Arechiga, "A hybrid model based on modular neural networks and fuzzy systems for classification of blood pressure and hypertension risk diagnosis,” Expert Systems with Applications, vol. 107, pp. 146$164,2018$.

[36] I. Miramontes, G. Martínez, P. Melin, and G. Prado-Arechiga, “A Hybrid Intelligent System Model for Hypertension Risk Diagnosis," Fuzzy Logic in Intelligent System Design, pp. 202-213, 2018.

[37] P. Melin, I. Miramontes, and G. Prado-Arechiga, "A new model for obtaining the risk assessment of developing hypertension based on artificial neural networks.," Journal of Hypertension, vol. 35, p. e210, 2017. 
[38] P. Melin, G. Prado-Arechiga, I. Miramontes, and J. C. Guzman, "Hypertension diagnosis with a soft computing model using a graphical user interface," Journal of Hypertension, vol. 37, p. e233, 2019.

[39] I. Miramontes, P. Melin, and G. Prado-Arechiga, "Particle Swarm Optimization of Modular Neural Networks for Obtaining the Trend of Blood Pressure," Intuitionistic and Type-2 Fuzzy Logic Enhancements in Neural and Optimization Algorithms: Theory and Applications, Eds. Cham: Springer International Publishing, 2020.

[40] I. Miramontes, P. Melin, and G. Prado-Arechiga, "Comparative Study of Bio-inspired Algorithms Applied in the Optimization of Fuzzy Systems," Hybrid Intelligent Systems in Control, Pattern Recognition and Medicine, O. Castillo and P. Melin, Eds. Cham: Springer International Publishing, 2020.

[41] C. J. Guzmán, I. Miramontes, P. Melin, and G. Prado-Arechiga, "Optimal Genetic Design of Type-1 and Interval Type-2 Fuzzy Systems for Blood Pressure Level Classification," Axioms, vol. 8, no. 1. pp. 1-35, 2019.

[42] J. C. Guzman, P. Melin, and G. Prado-Arechiga, "Design of an optimized fuzzy classifier for the diagnosis of blood pressure with a new computational method for expert rule optimization," Algorithms, vol. 10, no. 3, pp. 1-27, 2017. 